\title{
On the phylogeny of Mustelidae subfamilies: analysis of seventeen nuclear non-coding loci and mitochondrial complete genomes
}

$\mathrm{Li} \mathrm{Yu}^{1 *+}$, Dan Peng ${ }^{1+}$, Jiang Liu ${ }^{1+}$, Pengtao Luan ${ }^{1}$, Lu Liang ${ }^{2}$, Hang Lee ${ }^{3}$, Muyeong Lee ${ }^{3}$, Oliver A Ryder ${ }^{4}$ and Yaping Zhang ${ }^{1,2^{*}}$

\begin{abstract}
Background: Mustelidae, as the largest and most-diverse family of order Carnivora, comprises eight subfamilies. Phylogenetic relationships among these Mustelidae subfamilies remain argumentative subjects in recent years. One of the main reasons is that the mustelids represent a typical example of rapid evolutionary radiation and recent speciation event. Prior investigation has been concentrated on the application of different mitochondrial (mt) sequence and nuclear protein-coding data, herein we employ 17 nuclear non-coding loci (>15 kb), in conjunction with $\mathrm{mt}$ complete genome data (>16 kb), to clarify these enigmatic problems.

Results: The combined nuclear intron and $\mathrm{mt}$ genome analyses both robustly support that Taxidiinae diverged first, followed by Melinae. Lutrinae and Mustelinae are grouped together in all analyses with strong supports. The position of Helictidinae, however, is enigmatic because the $\mathrm{mt}$ genome analysis places it to the clade uniting Lutrinae and Mustelinae, whereas the nuclear intron analysis favores a novel view supporting a closer relationship of Helictidinae to Martinae. This finding emphasizes a need to add more data and include more taxa to resolve this problem. In addition, the molecular dating provides insights into the time scale of the origin and diversification of the Mustelidae subfamilies. Finally, the phylogenetic performances and limits of nuclear introns and $\mathrm{mt}$ genes are discussed in the context of Mustelidae phylogeny.

Conclusion: Our study not only brings new perspectives on the previously obscured phylogenetic relationships among Mustelidae subfamilies, but also provides another example demonstrating the effectiveness of nuclear noncoding loci for reconstructing evolutionary histories in a group that has undergone rapid bursts of speciation.
\end{abstract}

\section{Background}

The Mustelidae is the largest and most-diverse family of Carnivora with a distribution throughout all continents except Australia and Antarctica [1,2]. Recent classifications of the Mustelidae recognize up to eight subfamilies: Mustelinae, Galictinae, Helictidinae, Martinae, Melinae, Lutrinae, Mellivorinae, and Taxidiinae [3-5]. Phylogenetic relationships among these subfamlies have been hotly disputed in pioneer studies [3-15] and are not well established yet. The main problem is that the

\footnotetext{
* Correspondence: yuli1220@yahoo.com.cn; zhangyp@mail.kiz.ac.cn † Contributed equally

${ }^{1}$ Laboratory for Conservation and Utilization of Bio-resource \& Key Laboratory for Microbial Resources of the Ministry of Education, Yunnan University, Kunming, 650091, PR, China

Full list of author information is available at the end of the article
}

family Mustelidae represents a typical example of rapid evolutionary radiation and recent speciation event $[6,7]$, dating back to the Oligocene [16-18]. For this reason, attempts to clarify relationships among the eight Mustelidae subfamilies based on a variety of molecular studies have encountered challenges.

Previously, molecular studies of the phylogenetic reconstruction of subfamilies within the Mustelidae were based on short fragments of nuclear and mt DNA. Recently, some efforts have been made to obtain the phylogenetic tree based on large datasets, including those using $12 \mathrm{mt}$ protein-coding genes [15], 5 nuclear genes [4,12], 4 nuclear genes and $1 \mathrm{mt}$ gene [10,14], 21 nuclear genes and $1 \mathrm{mt}$ gene [5] and 25 nuclear genes and $\mathrm{mt}$ genes [13]. Despite numerous efforts, however, 

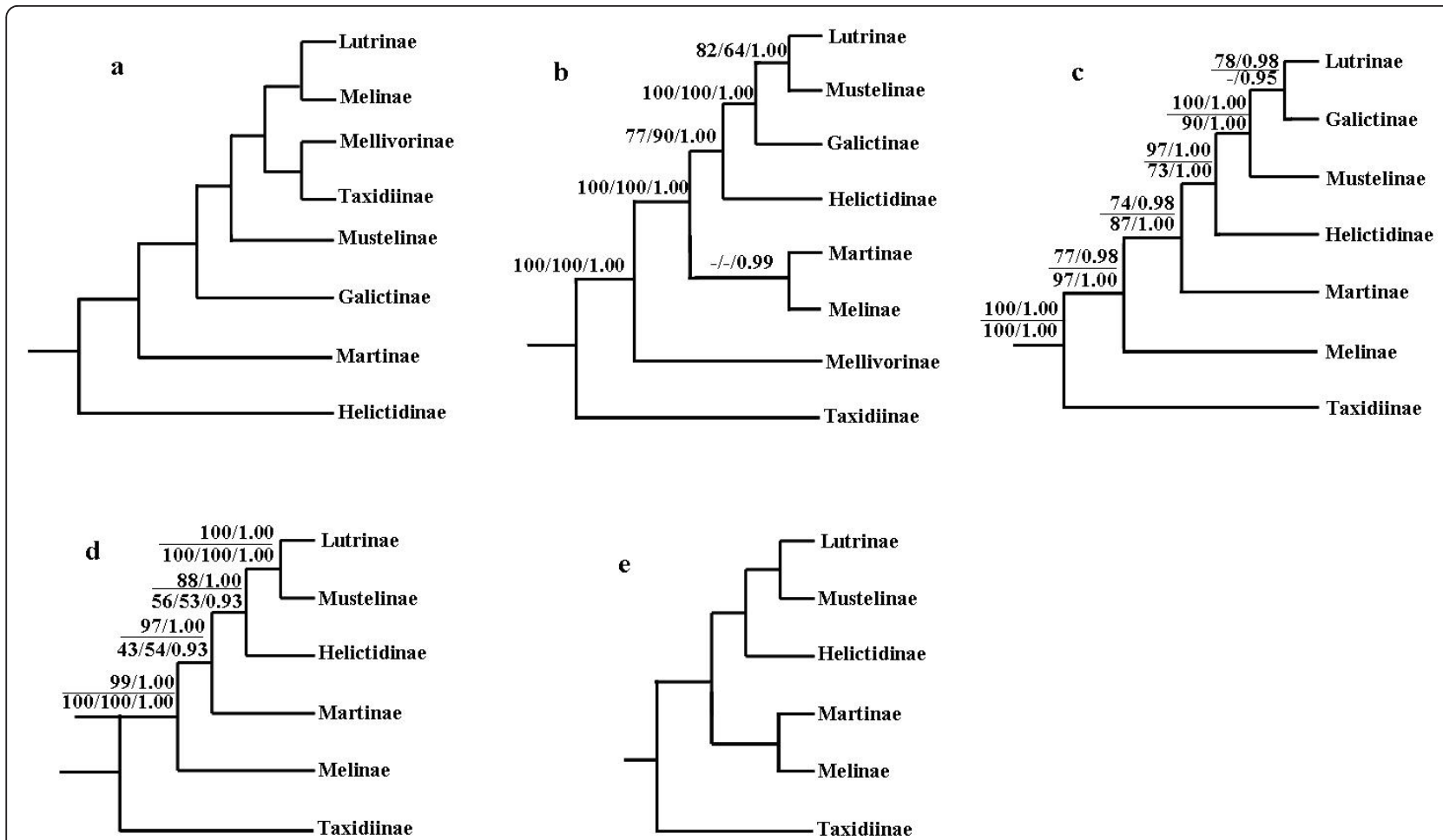

Figure 1 Hypotheses of phylogenetic relationships among Mustelidae subfamilies. Trees were reconstructed based on (a) 46 morphological characters [8], (b) analyses of 21 nuclear genes and $1 \mathrm{mt}$ gene [5], (c) analyses of 5 nuclear genes and $1 \mathrm{mt}$ gene [10] (support values are indicated above the line) and 25 nuclear genes and mt genome [13] (support values are indicated below the line), (d) analyses of 5 nuclear genes [4] (support values are indicated above the line) [12] support values are indicated below the line), (e) supertree analyses of 5 nuclear genes [4].

evolutionary relationships among the Mustelidae subfamilies remain controversial (see Figure 1).

Hence, it is necessary to exploit larger independent sources of phylogenetic characters to clarify these enigmatic problems. Several studies have shown that relative to the commonly used nuclear protein-coding and $\mathrm{mt}$ genes, the noncoding intron sequences can be an equally fruitful source of phylogenetic characters as they possess a number of traits that are desirable for molecular phylogenetics [19-24], for example, lack of functional constraints, a high substitution rate and less homoplasy $[19,25,26]$. In these studies, the nuclear introns have been shown to provide powerful complementary data to address the ambiguous relationships of different taxonomic levels, including the beaked whale species [23], the Asian pitvipers genus [19], the carnivoran families $[24,27]$, and the eutherian orders [21].

In the present study, we aim to sequence 17 nuclear intron loci comprising a total of $>15 \mathrm{~kb}$ from 17 mustelids. The mustelids examined here represent all subfamilies of Mustelidae, except for Mellivorinae and Galictinae. Of the 17 nuclear loci, 14 were first applied in the studies of Mustelidae phylogeny. In addition, we undertook the sequencing of the $\mathrm{mt}$ genome from these species and presented a phylogeny based on the mt genome data currently available for mustelids. Our objectives were to: (1) provide new insights into the relationships among the Mustelidae subfamilies, and (2) examine the utilities and evolutionary dynamics of the nuclear and $\mathrm{mt}$ genes in the context of Mustelidae phylogeny, with special attention to the previously unexplored nuclear intron genes.

\section{Results}

\section{Characteristics of the Nuclear Intron Data and Mt} Genomes

The general characteristics of the nuclear intron data and $\mathrm{mt}$ genomes are summarized in Table 1 . The 17 nuclear introns of 21 species varied in length from 642 (Fgb-7) to 1685 (Plod2-14) aligned positions. The removal of ambiguous areas resulted in length variation of the aligned sequences from 523 (Fgb-4) to 1332 (Coro1c-4) positions. The numbers of parsimony-informative sites range from $71(13.58 \%)(F g b-4)$ to 343 (30.25\%) (Cidea-1). According to different gap selection criteria in Gblocks, the alignment of the combined dataset comprised 15688 (allowed gap positions = all), 15038 (allowed gap positions $=$ with half) and 12570 (allowed 
Table 1 Characterization of Nuclear introns and $\mathrm{mt}$ genes examined in the present study

\begin{tabular}{|c|c|c|c|c|c|c|c|c|c|c|c|c|c|c|}
\hline \multirow[t]{2}{*}{$\begin{array}{l}\text { Sequence } \\
\text { type }\end{array}$} & \multirow[t]{2}{*}{$\begin{array}{c}\text { Fragments } \\
\text { name }\end{array}$} & \multirow[t]{2}{*}{ TEs } & \multirow[t]{2}{*}{ Aligned $^{a}$} & \multirow[t]{2}{*}{$\begin{array}{l}\text { Final } \\
\text { data }^{b}\end{array}$} & \multirow[t]{2}{*}{$\begin{array}{c}\text { Parsimony- } \\
\text { informative sites }\end{array}$} & \multicolumn{4}{|c|}{ Nucleotide Composition } & \multirow[t]{2}{*}{$\begin{array}{l}\text { Ti/ } \\
\text { Tv }\end{array}$} & \multirow[t]{2}{*}{$\begin{array}{l}\text { Best fit } \\
\text { model }\end{array}$} & \multicolumn{2}{|c|}{$\begin{array}{l}\text { Among- } \\
\text { site Rate } \\
\text { Variation }\end{array}$} & \multirow[t]{2}{*}{$\begin{array}{c}\text { Pairwise } \\
\text { Distance (\%) }\end{array}$} \\
\hline & & & & & & A & $T$ & G & C & & & 1 & $\alpha$ & \\
\hline \multirow{20}{*}{$\begin{array}{l}\text { Nucear } \\
\text { introns }\end{array}$} & Cidea-1 & YES & 1590 & 1134 & 343 & 0.308 & 0.240 & 0.230 & 0.222 & 1.5 & $\mathrm{TrN}+\mathrm{G}$ & 0 & 0.9770 & 7.5 \\
\hline & Coro1c-4 & YES & 1435 & 1332 & 339 & 0.242 & 0.280 & 0.234 & 0.243 & 2.4 & $K 81 u f+G$ & 0 & 0.9379 & 6.3 \\
\hline & Coro1c-5 & YES & 1380 & 1099 & 140 & 0.309 & 0.321 & 0.210 & 0.160 & 2.2 & $\mathrm{TVM}+\mathrm{G}$ & 0 & 0.9084 & 4.7 \\
\hline & Guca1b-3 & YES & 711 & 711 & 149 & 0.230 & 0.186 & 0.289 & 0.295 & 1.6 & $H K Y+G$ & 0 & 0.7174 & 6.1 \\
\hline & Ociad1-4 & YES & 1576 & 1014 & 200 & 0.328 & 0.393 & 0.145 & 0.134 & 1.9 & $\mathrm{TVM}+\mathrm{G}$ & 0 & 1.3754 & 4.1 \\
\hline & Plod2-13 & NO & 1317 & 1230 & 236 & 0.293 & 0.325 & 0.229 & 0.153 & 2.0 & $\mathrm{GTR}+\mathrm{G}$ & 0 & 1.555 & 3.3 \\
\hline & Plod2-14 & YES & 1685 & 1068 & 263 & 0.342 & 0.327 & 0.180 & 0.151 & 1.4 & TVM+G & 0 & 1.4866 & 3.7 \\
\hline & Ssr1-5 & YES & 1180 & 783 & 131 & 0.324 & 0.382 & 0.160 & 0.134 & 2.2 & $\mathrm{GTR}+\mathrm{G}$ & 0 & 1.4043 & 3.6 \\
\hline & Tbc1d7-6 & YES & 1153 & 1095 & 326 & 0.247 & 0.293 & 0.261 & 0.199 & 1.5 & $\mathrm{TIM}+\mathrm{G}$ & 0 & 0.7365 & 8.2 \\
\hline & Tinagl1-1 & YES & 1263 & 1260 & 250 & 0.208 & 0.208 & 0.295 & 0.290 & 2.4 & $\mathrm{HKY}+\mathrm{G}$ & 0 & 0.7637 & 3.2 \\
\hline & Tinagl1-3 & YES & 1115 & 1014 & 193 & 0.179 & 0.238 & 0.281 & 0.301 & 1.9 & K81uf+G & 0 & 0.7201 & 3.7 \\
\hline & Wasf1-3 & YES & 1195 & 1021 & 161 & 0.314 & 0.345 & 0.170 & 0.171 & 1.8 & $\mathrm{GTR}+\mathrm{G}$ & 0 & 1.6253 & 3.9 \\
\hline & Wasf1-6 & YES & 1276 & 1077 & 207 & 0.308 & 0.354 & 0.191 & 0.147 & 2.2 & $\mathrm{TVM}+\mathrm{G}$ & 0 & 1.7531 & 4.1 \\
\hline & Wasf1-7 & NO & 1380 & 1147 & 223 & 0.314 & 0.355 & 0.165 & 0.166 & 2.0 & $\mathrm{GTR}+\mathrm{G}$ & 0 & 0.6571 & 3 \\
\hline & Ttr-1 & YES & 1130 & 895 & 159 & 0.263 & 0.279 & 0.227 & 0.231 & 2.4 & $T V M+G$ & 0 & 1.3403 & 4.6 \\
\hline & Fgb-4 & YES & 847 & 523 & 71 & 0.307 & 0.307 & 0.215 & 0.171 & 2.0 & HKY & 0 & equal & 4.5 \\
\hline & Fgb-7 & YES & 642 & 611 & 102 & 0.292 & 0.327 & 0.186 & 0.195 & 1.4 & GTR & 0 & equal & 5.1 \\
\hline & $\overline{\mathrm{Com} 1^{\mathrm{C}}}$ & & 15893 & 12570 & 1735 & 0.279 & 0.301 & 0.219 & 0.201 & 2.0 & $T V M+I+G$ & 0.17 & 0.9090 & 4.4 \\
\hline & $\mathrm{Com} 2^{c}$ & & 15893 & 15038 & 2137 & 0.279 & 0.302 & 0.218 & 0.201 & 1.9 & $\mathrm{GTR}+\mathrm{I}+\mathrm{G}$ & 0.18 & 0.9078 & 4.6 \\
\hline & $\mathrm{Com}^{\mathrm{c}}$ & & 15893 & 15688 & 2170 & 0.279 & 0.303 & 0.218 & 0.200 & 1.8 & $\mathrm{GTR}+\mathrm{I}+\mathrm{G}$ & 0.18 & 0.9052 & 4.6 \\
\hline \multirow[t]{18}{*}{ Mt genes } & ND1 & & 957 & & 357 & 0.309 & 0.274 & 0.122 & 0.295 & 2.6 & $\mathrm{GTR}+\mathrm{I}+\mathrm{G}$ & 0.53 & 0.7988 & 17.3 \\
\hline & ND2 & & 1044 & & 483 & 0.363 & 0.263 & 0.098 & 0.275 & 2.1 & $\mathrm{GTR}+\mathrm{I}+\mathrm{G}$ & 0.35 & 0.859 & 23 \\
\hline & $\operatorname{cox} 1$ & & 1545 & & 556 & 0.282 & 0.302 & 0.175 & 0.241 & 3.1 & $\mathrm{GTR}+\mathrm{I}+\mathrm{G}$ & 0.58 & 1.1772 & 19.1 \\
\hline & $\operatorname{cox} 2$ & & 684 & & 252 & 0.323 & 0.276 & 0.142 & 0.260 & 3.3 & $\begin{array}{c}\text { K81uf }+ \text { I } \\
+G\end{array}$ & 0.56 & 1.0711 & 19.6 \\
\hline & ATP8 & & 204 & & 92 & 0.388 & 0.291 & 0.077 & 0.243 & 2.2 & $\mathrm{GTR}+\mathrm{I}+\mathrm{G}$ & 0.42 & 1.3437 & 22.8 \\
\hline & ATP6 & & 681 & & 290 & 0.306 & 0.291 & 0.116 & 0.287 & 3.3 & $\mathrm{GTR}+\mathrm{I}+\mathrm{G}$ & 0.46 & 0.7582 & 21.6 \\
\hline & $\operatorname{cox} 3$ & & 784 & & 303 & 0.273 & 0.286 & 0.154 & 0.286 & 2.6 & $\operatorname{TrN}+\mathrm{I}+\mathrm{G}$ & 0.53 & 0.8079 & 20.2 \\
\hline & ND3 & & 348 & & 160 & 0.320 & 0.279 & 0.130 & 0.272 & 2.5 & $\operatorname{TrN}+\mathrm{I}+\mathrm{G}$ & 0.38 & 0.956 & 22.3 \\
\hline & ND4L & & 297 & & 127 & 0.287 & 0.324 & 0.124 & 0.265 & 2.9 & TVM+I+G & 0.43 & 0.7530 & 22.5 \\
\hline & ND4 & & 1378 & & 609 & 0.323 & 0.278 & 0.115 & 0.284 & 2.8 & $\mathrm{GTR}+\mathrm{I}+\mathrm{G}$ & 0.42 & 0.835 & 22.1 \\
\hline & ND5 & & 1830 & & 739 & 0.331 & 0.274 & 0.115 & 0.280 & 2.6 & $\mathrm{TIM}+\mathrm{I}+\mathrm{G}$ & 0.42 & 0.8367 & 19.6 \\
\hline & ND6 & & 534 & & 192 & 0.417 & 0.203 & 0.101 & 0.278 & 2.7 & $\begin{array}{c}\text { K81uf+l } \\
+G\end{array}$ & 0.46 & 0.656 & 16.3 \\
\hline & CYTB & & 1140 & & 446 & 0.295 & 0.278 & 0.134 & 0.293 & 2.8 & $T V M+I+G$ & 0.5 & 1.1027 & 18.7 \\
\hline & 12SrRNA & & 988 & & 236 & 0.371 & 0.229 & 0.178 & 0.223 & 2.8 & $\mathrm{GTR}+\mathrm{I}+\mathrm{G}$ & 0.37 & 0.3532 & 9.2 \\
\hline & 16SrRNA & & 1626 & & 403 & 0.369 & 0.243 & 0.174 & 0.213 & 2.1 & $\mathrm{GTR}+\mathrm{I}+\mathrm{G}$ & 0.47 & 0.5765 & 10.5 \\
\hline & tRNA & & 1558 & & 301 & 0.353 & 0.281 & 0.157 & 0.209 & 4.0 & $\mathrm{GTR}+\mathrm{I}+\mathrm{G}$ & 0.48 & 0.4371 & 7.3 \\
\hline & D-loop & & 941 & & 315 & 0.308 & 0.277 & 0.166 & 0.249 & 1.9 & $\mathrm{HKY}+\mathrm{I}+\mathrm{G}$ & 0.3 & 0.4867 & 11.4 \\
\hline & Combined & & 16537 & & 5855 & 0.329 & 0.272 & 0.140 & 0.259 & 2.7 & $G T R+I+G$ & 0.5 & 0.9372 & 16.9 \\
\hline
\end{tabular}

Note: $\mathrm{Ti}=$ Transition; Tv = Transversion; I = Proportion of invariable sites; $\alpha=$ Gamma distribution shape parameter; TEs = Transposible Elements. If TEs were detected in introns, it indicated YES, otherwise it indicated NO.

a The length of sequences which were aligned using the CLUSTAL software with default settings.

$\mathrm{b}$ The length of analyzed data, after the ambiguous areas of the alignment were removed by Gblocks $0.91 \mathrm{~b}$.

c The length of all introns concatenated, after the ambiguous areas of the alignment were removed by Gblocks $0.91 \mathrm{~b}$ with no gap (Com1), half gap (Com2), and all gap (Com3) parameters. 
gap positions $=$ none ) positions. The parsimony-informative sites in these three datasets are 2170 (13.83\%), 2137(14.21\%) and 1735 (13.80\%), respectively. An A-T bias (average $=58.64 \%$ ) and low transition $(\mathrm{Ti}) /$ transversion $(\mathrm{Tv})$ rate ratio (average $=1.93$ ) were observed in most introns. In addition, most introns showed gamma shape parameters $(\alpha)$ close to or larger than 1.0. The nuclear sequence divergence among ingroup taxa ranged from 3\% (Wasf1-7) to $8.2 \%$ (Tbc1d7-6), and averaged $4.7 \%$.

The complete mt genomes of 25 species ranged from 16388 to $16623 \mathrm{bp}$ in size. Length differences are largely due to the variation in tandem repeats within the control region. All genomes shared the same 13 proteincoding genes, 22 tRNAs genes, 2 rRNAs, and a control region, and also the same gene order. These mt genomes are apparently AT-biased (average $=60.1 \%$ ). The sequence divergence among ingroup taxa ranged from 16.3 (ND6) to 23\% (ND2) for the protein-coding dataset (average 20.4\%), from 9.2 (12S rRNA) to $10.5 \%$ (16S $r R N A$ ) for the rRNA dataset (average 9.85\%), 7.3\% for the $t R N A$ dataset, $11.4 \%$ for the control region, and $16.6 \%$ for the complete dataset.

\section{Occurrence of Transposable Elements (TEs)}

In our intron datasets, pervasive transposable element (TE) insertions were discovered (Table 2), which are mainly non-long-terminal repeat retrotransposons (nonLTR), e.g., long interspersed elements (LINEs), short interspersed elements (SINEs), mammalian-wide interspersed repeats (MIRs), and DNA transposons. MIRs and DNA transposons integrated into the orthologous loci of all examined species, which suggested an ancient origin. The result was consistent with the earlier finding that these two classes of TEs represented remnants or "fossils" of TEs, predating the radiation of mammalian orders, and had long ago become inactive in mammalian lineages [28-30].

The great majority of LINEs and SINEs identified here were members of the L1_Canid (Fc) and CAN SINE groups that have been exclusively found in Carnivora [31-38]. Most of SINEs showed restricted taxonomic distributions and were characterized by sporadic locations in the intronic regions. This suggests that those SINEs emerged after species diversification, likely retaining their ability to retrotranspose.

The insertions of TEs at genomic sites are often considered irreversible and random [39], which suggests that they may be excellent homoplasy-free markers in phylogenetic analyses [40-43]. Here, we identified one SINE insertion shared by Martes flavigula, Martes zibellina, Martes foina, Martes pennanti, Martes amaricana, and Gulo gulo, supporting their close relationship and the monophyly of Martinae subfamily.
Occurrence of Intra-individual Allele Heterozygotes (IIAHs) The overall incidence of intra-individual allele heterozygotes (IIAHs) in our 14 new introns appears universal (Table 3). There were 106 cases of IIAHs in total. Of the 21 species examined, 3 to 11 cases of IIAHs were detected from each intron. IIAHs were observed to be either of equal or variable length. 11 of 14 introns had allele length variant heterozygotes due to a 1-bp indel, with the other nucleotide sites either the same or distinct at 1-11 bp. IIAHs of identical length were discovered in all introns with 1-10 substitutional differences.

Generally, IIAHs formed monophyletic pairs on the phylogenetic trees as expected (see Additional file 1). Two cases of strongly-supported nonmonophyletic IIAHs were illustrated by the close relatedness of one allele of the least weasel Mustela nivalis to one allele of the European polecat Mustela putorius (Plod2-13 and Guca1b-3 genes; Figure 2), which are most likely to indicate the cases of incomplete lineage sorting.

\section{Phylogenetic Inference}

Although individual nuclear gene analyses produced inconsistent topologies with low levels of support (Additional file 1), possibly due to limited phylogenetic information harbored in a single gene, the analyses of the combined nuclear data set using three gap selection criteria in Gblocks (allowed gap positions = none, with half, and all) and different tree-building methods (MP, ML and Bayesian methods) yielded nearly identical, well-resolved trees with strong support for all nodes, except for the relationships among Gulo gulo, Martes americana, and Martes pennanti (Figure 2). In the tree, Taxidiinae diverged first (MP $\mathrm{BS}=100 \%, \mathrm{ML} \mathrm{BS}=100 \%, \mathrm{PP}=1.00)$, followed by Melinae $(\mathrm{MP} B S=85 \%, \mathrm{ML} \mathrm{BS}=98 \%, \mathrm{PP}=1.00)$. The remaining mustelids were divided into two clades, one consisting of Martinae and Helictidinae (MP BS = $99 \%, \mathrm{ML} B S=99 \%, \mathrm{PP}=1.00)$, and the other one consisting of Lutrinae and Mustelinae (MP BS $=82 \%$, $\mathrm{ML} \mathrm{BS}=99 \%, \mathrm{PP}=1.00)$. In addition, both the inclusion of IIAHs in the combined nuclear analysis using software POFAD [44] and the Bayesian concordance analysis (BCA) analysis (Additional file 2) using software BUCKy [45] produced the same tree topologies as that in Figure 2. At the subfamily level, all nodes in the BCA analysis received high concordance factors (CF) value (1.000).

For individual mt gene analyses, the rRNA and tRNA data sets demonstrated reduced resolving power for phylogenetic inference compared to protein-coding gene analysis (Additional file 3). The complete mtDNA genome-based analyses, irrespective of the used tree-building methods and parameter sets, produced a well-resolved and well-supported tree (Figure 3), with the tree topology 
Table 2 Transposable Elements (TEs) discovered in the present study

\begin{tabular}{|c|c|c|c|c|c|c|c|}
\hline \multirow{3}{*}{$\begin{array}{c}\text { Intron } \\
\text { Fragments }\end{array}$} & \multirow[t]{3}{*}{ Species } & \multicolumn{6}{|c|}{ Transposable Elements (TEs) } \\
\hline & & \multicolumn{3}{|c|}{ Species-specific } & \multicolumn{3}{|c|}{ Orthologous } \\
\hline & & TEs & Class & $\begin{array}{l}\text { Length } \\
\text { (bp) }\end{array}$ & TEs & Class & $\begin{array}{l}\text { Length } \\
\text { (bp) }\end{array}$ \\
\hline \multirow[t]{4}{*}{ Cidea1 } & Martes penanti & SINEC_b2 & SINE/tRNA-Lys & 192 & L1_Canid_ & LINE/L1 & $54-82$ \\
\hline & Mephitis mephitis & SINEC_b1 & SINE/tRNA-Lys & 188 & & & \\
\hline & Meles meles & SINEC_b1 & SINE/tRNA-Lys & 194 & & & \\
\hline & Arctonyx collaris & SINEC_b2 & SINE/tRNA-Lys & 194 & & & \\
\hline \multirow[t]{2}{*}{ Corolc-4 } & & & & & Tigger12c & $\begin{array}{l}\text { DNA/TcMar- } \\
\text { Tigger }\end{array}$ & $56-59$ \\
\hline & & & & & $\mathrm{MIRb}$ & SINE/MIR & $93-112$ \\
\hline \multirow[t]{2}{*}{ Corolc-5 } & Lutra lutra & SINEC_b1 & SINE/tRNA-Lys & 187 & SINEC_old & SINE/tRNA-Lys & $104-107$ \\
\hline & & & & & L1ME4a & LINE/L1 & $65-67$ \\
\hline Guca1b-3 & & & & & MIR & SINE/MIR & $58-63$ \\
\hline \multirow[t]{2}{*}{ Ociad1-4 } & Mephitis mephitis & SINEC_b2 & SINE/tRNA-Lys & 268 & MIRC & SINE/MIR & $92-122$ \\
\hline & $\begin{array}{l}\text { Martes flavigula/Martes zibellina/Gulo } \\
\text { gulo/Martes foina/Martes amaricana/Martes } \\
\text { penanti }\end{array}$ & SINEC_b1 & SINE/tRNA-Lys & 175-187 & & & \\
\hline \multirow[t]{2}{*}{ Plod2-14 } & Raccoon/Kinkajou & SINEC_b1 & SINE/tRNA-Lys & 192-195 & Kanga1a & DNA/TcMar-Tc2 & $129-188$ \\
\hline & & & & & Kanga1c & DNA/TcMar-Tc2 & $81-92$ \\
\hline Ssr1-5 & Ailurus fulgens & SINEC_b1 & SINE/tRNA-Lys & 196 & & & \\
\hline Tbc1d7-6 & & & & & MIR & SINE/MIR & 134-195 \\
\hline Tinagl1-1 & Mephitis mephitis/Ailurus fulgens & MIR3 & SINE/MIR & $72-76$ & MIR & SINE/MIR & $49-64$ \\
\hline Tinagl1-3 & & & & & MIR & SINE/MIR & $85-87$ \\
\hline Wasf1-3 & & & & & $\mathrm{L} 2 \mathrm{C}$ & LINE/L2 & $85-90$ \\
\hline Wasf1-6 & & & & & MER58A & $\begin{array}{l}\text { DNA/hAT- } \\
\text { Charlie }\end{array}$ & 197 \\
\hline \multirow[t]{2}{*}{ Fgb-4 } & Taxidea taxus & SINEC_b1 & SINE/tRNA-Lys & 199 & & & \\
\hline & Lutra lutra & SINEC_b2 & SINE/tRNA-Lys & 187 & & & \\
\hline \multirow[t]{2}{*}{ Fgb-7 } & Mephitis mephitis & SINEC_b1 & SINE/tRNA-Lys & 193 & $\mathrm{MIRb}$ & SINE/MIR & $196-198$ \\
\hline & Mephitis mephitis & SINEC_b2 & SINE/tRNA-Lys & 193 & & & \\
\hline Ttr-1 & & & & & SINEC_b1 & SINE/tRNA-Lys & $166-193$ \\
\hline
\end{tabular}

and branch supports identical to that of the combined protein-coding gene analysis. At the subfamilial level, the single difference between $\mathrm{mt}$ genome tree (Figure 3) and combined nuclear gene tree (Figure 2) is the phylogenetic position of Helictidinae. In mt genome tree, Helictidinae is closer to the clade uniting Lutrinae and Mustelinae than to Martinae ( $\mathrm{MP}=60 \%$; $\mathrm{ML} \mathrm{BS}=95 \%$; $\mathrm{PP}=1.00$ ). The phylogenetic tree reconstructed from the combined nuclear and $\mathrm{mt}$ genome data set by using the BCA analysis [45] produced a tree topology (Additional file 2) identical to that from the combined nuclear gene analysis (Figure 2). At the subfamily level, all nodes received high CFs (1.000), except for that of Helictidinae (CF $=$ 0.502 ), whose position is the single discrepancy between the combined nuclear and $\mathrm{mt}$ gene trees.

Shimodaira-Hasegawa (SH) test and the approximately unbiased (AU) test were carried out to examine the degree of significant difference between the nuclear and mt trees produced in the present study. Both tests indicated significant topological incongruence concerning the phylogenetic position of Helictidinae between the nuclear and $\mathrm{mt}$ trees. When using nuclear data, the $\mathrm{mt}$ genome tree topology, in which Helictidinae is closer to the clade uniting Lutrinae and Mustelinae, was rejected by the AU and SH tests $(P<0.05)$. When using $\mathrm{mt}$ genome data, the nuclear tree topology, in which Helictidinae and Martinae are grouped together, was rejected by the AU and SH tests $(P<0.05)$.

\section{Divergence Time Estimation}

Divergence time estimates for the origin and diversification of Mustelidae subfamilies yielded broadly consistent results between combined nuclear and $\mathrm{mt}$ genome data set (Table 4). Combined nuclear gene analysis placed the earliest branching Taxidiinae around 23.73 Mya (95\% confidence intervals $=22.80-24.70 \mathrm{Mya})$. After 


\begin{tabular}{|c|c|c|c|c|c|c|c|c|c|c|c|c|c|c|c|c|c|c|c|c|c|}
\hline \multirow[t]{2}{*}{ Introns } & \multicolumn{21}{|c|}{ Species } \\
\hline & $\begin{array}{l}\text { Mephitis } \\
\text { mephitis }\end{array}$ & $\begin{array}{l}\text { Ailurus } \\
\text { fulgens }\end{array}$ & $\begin{array}{l}\text { Procyon } \\
\text { lotor }\end{array}$ & $\begin{array}{l}\text { Potos } \\
\text { flavus }\end{array}$ & $\begin{array}{c}\text { Martes } \\
\text { flavigula }\end{array}$ & $\begin{array}{c}\text { Martes } \\
\text { zibellina }\end{array}$ & $\begin{array}{l}\text { Martes } \\
\text { foina }\end{array}$ & $\begin{array}{c}\text { Martes } \\
\text { amaricana }\end{array}$ & $\begin{array}{l}\text { Martes } \\
\text { penanti }\end{array}$ & $\begin{array}{l}\text { Gulo } \\
\text { gulo }\end{array}$ & $\begin{array}{l}\text { Mustela } \\
\text { kathiah }\end{array}$ & $\begin{array}{c}\text { Mustela } \\
\text { nivalis }\end{array}$ & $\begin{array}{l}\text { Mustela } \\
\text { sibirica }\end{array}$ & $\begin{array}{l}\text { Mustela } \\
\text { frenata }\end{array}$ & $\begin{array}{l}\text { Mustela } \\
\text { putorius }\end{array}$ & $\begin{array}{l}\text { Mustela } \\
\text { vison }\end{array}$ & $\begin{array}{c}\text { Arctonyx } \\
\text { collaris }\end{array}$ & $\begin{array}{l}\text { Meles } \\
\text { meles }\end{array}$ & $\begin{array}{l}\text { Melogale } \\
\text { moschata }\end{array}$ & $\begin{array}{l}\text { Taxidea } \\
\text { taxus }\end{array}$ & $\begin{array}{l}\text { Lutra } \\
\text { lutra }\end{array}$ \\
\hline Cidea-1 & $0 / 1 / 0$ & 0/0/0 & $0 / 1 / 0$ & 0/1/0 & 0/0/0 & $0 / 3 / 0$ & 0/0/0 & 0/0/0 & 0/0/0 & - & 0/0/0 & 0/0/0 & $1 / 1 / 1$ & 0/0/0 & 0/0/0 & 0/0/0 & $0 / 3 / 1$ & $0 / 2 / 0$ & $0 / 1 / 0$ & 0/0/0 & - \\
\hline $\begin{array}{l}\text { Corole- } \\
4\end{array}$ & $3 / 1 / 0$ & $3 / 1 / 0$ & 0/0/0 & 0/1/0 & 0/0/0 & $2 / 0 / 1$ & 0/0/0 & 0/0/0 & 0/0/0 & 0/0/0 & 0/0/0 & $2 / 0 / 0$ & - & 0/0/0 & - & 0/0/0 & 0/0/0 & $4 / 6 / 0$ & 0/0/0 & 0/0/0 & $2 / 0 / 0$ \\
\hline $\begin{array}{l}\text { Coro1c- } \\
5\end{array}$ & 0/0/0 & 0/0/0 & 0/0/0 & 0/0/0 & 0/0/0 & 0/0/0 & 0/1/0 & - & - & - & 0/0/0 & $0 / 5 / 0$ & 0/0/0 & - & $0 / 5 / 0$ & - & 0/0/0 & 0/0/0 & - & $0 / 1 / 0$ & $0 / 0 / 0$ \\
\hline $\begin{array}{l}\text { Gucalb- } \\
3\end{array}$ & 0/0/0 & 0/0/0 & $0 / 0 / 1$ & - & $5 / 0 / 0$ & 0/0/0 & 0/0/0 & 0/0/0 & $4 / 0 / 0$ & 0/0/0 & 0/0/0 & $3 / 0 / 1$ & $0 / 2 / 0$ & 0/0/0 & $2 / 0 / 0$ & 0/0/0 & $4 / 6 / 0$ & $0 / 1 / 0$ & 0/0/0 & $1 / 0 / 0$ & $0 / 1 / 0$ \\
\hline $\begin{array}{l}\text { Ociad1- } \\
4\end{array}$ & 0/0/0 & 0/0/0 & $0 / 3 / 0$ & 0/1/1 & $1 / 0 / 1$ & $0 / 1 / 0$ & $1 / 1 / 0$ & 0/0/0 & 0/0/0 & 0/0/0 & $1 / 4 / 0$ & 0/0/0 & 0/0/0 & 0/0/0 & 0/0/0 & 0/0/0 & $0 / 1 / 0$ & 0/0/0 & 0/0/0 & $1 / 1 / 0$ & 0/0/0 \\
\hline $\begin{array}{l}\text { Plod2- } \\
13\end{array}$ & 0/0/0 & $1 / 1 / 0$ & 0/0/0 & $4 / 1 / 1$ & 0/0/1 & $2 / 1 / 0$ & 0/0/0 & 0/0/0 & 0/0/0 & 0/0/0 & $1 / 0 / 0$ & 0/0/0 & 0/0/0 & $1 / 0 / 0$ & $1 / 0 / 0$ & 0/0/0 & 0/0/0 & $1 / 5 / 0$ & $3 / 2 / 0$ & 0/0/0 & $0 / 1 / 0$ \\
\hline $\begin{array}{l}\text { Plod2- } \\
14\end{array}$ & $0 / 1 / 1$ & 0/0/0 & 0/0/0 & $1 / 6 / 1$ & $0 / 3 / 0$ & 0/1/0 & 0/1/0 & 0/0/0 & 0/0/0 & 0/0/0 & 0/0/0 & $1 / 1 / 0$ & $1 / 0 / 0$ & 0/0/0 & 0/0/0 & 0/0/0 & 0/0/0 & 0/0/0 & 0/0/0 & 0/0/0 & 0/0/0 \\
\hline Ssr1-5 & 0/0/0 & 0/0/0 & $0 / 1 / 1$ & 0/0/1 & 0/0/0 & $0 / 2 / 0$ & 0/3/0 & 0/0/0 & 0/0/0 & 0/0/0 & $0 / 1 / 0$ & 0/0/0 & 0/0/0 & 0/0/0 & $1 / 3 / 0$ & 0/0/0 & 0/0/0 & 0/0/0 & 0/0/0 & $0 / 2 / 0$ & 0/0/0 \\
\hline $\begin{array}{l}\text { Tbc1d7- } \\
6\end{array}$ & $1 / 2 / 1$ & 0/0/1 & 0/0/0 & $2 / 1 / 0$ & $1 / 0 / 0$ & $1 / 2 / 0$ & $4 / 2 / 0$ & 0/0/0 & 0/0/0 & 0/0/0 & $1 / 0 / 0$ & $7 / 4 / 1$ & 0/0/0 & $1 / 0 / 0$ & $1 / 0 / 0$ & 0/0/0 & 0/0/0 & 0/0/0 & 0/0/0 & $0 / 1 / 0$ & 0/0/0 \\
\hline $\begin{array}{l}\text { Tinag1- } \\
1\end{array}$ & 0/0/0 & 0/0/0 & 0/0/0 & $3 / 0 / 0$ & $2 / 1 / 1$ & $3 / 1 / 0$ & 0/0/0 & 0/0/0 & $1 / 0 / 0$ & 0/0/0 & $3 / 0 / 0$ & $2 / 0 / 0$ & 0/0/0 & 0/0/0 & $3 / 0 / 0$ & 0/0/0 & 0/0/0 & 0/0/0 & $3 / 0 / 0$ & 0/0/0 & $1 / 0 / 0$ \\
\hline $\begin{array}{l}\text { Tinagl1- } \\
3\end{array}$ & 0/0/0 & 0/1/0 & 0/0/0 & $1 / 6 / 0$ & $1 / 0 / 0$ & $0 / 1 / 0$ & 0/0/0 & 0/0/0 & 0/0/0 & 0/0/0 & $0 / 5 / 0$ & 0/0/0 & 0/0/0 & 0/0/0 & 0/0/0 & 0/0/0 & $1 / 1 / 0$ & $1 / 0 / 0$ & $1 / 0 / 0$ & 0/0/0 & - \\
\hline Wasf1-3 & 0/0/0 & 0/0/0 & 0/0/0 & $2 / 0 / 1$ & 0/0/0 & $0 / 2 / 0$ & 0/0/0 & 0/0/0 & 0/0/0 & 0/0/0 & 0/0/0 & $0 / 3 / 0$ & $2 / 0 / 0$ & $1 / 0 / 0$ & 0/0/0 & 0/0/0 & 0/0/0 & 0/0/0 & 0/0/0 & 0/0/0 & 0/0/0 \\
\hline Wasf1-6 & 0/0/0 & 0/0/0 & $3 / 1 / 0$ & $2 / 0 / 0$ & 0/0/0 & 0/0/0 & 0/0/0 & - & 0/0/0 & 0/0/0 & 0/0/0 & 0/0/0 & 0/0/0 & 0/0/0 & 0/0/0 & 0/0/0 & 0/0/0 & 0/0/0 & 0/0/0 & $1 / 0 / 0$ & $0 / 0 / 0$ \\
\hline Wasf1-7 & 0/0/1 & $1 / 0 / 1$ & $2 / 0 / 0$ & $1 / 1 / 0$ & $1 / 0 / 0$ & 0/0/0 & $1 / 0 / 0$ & 0/0/0 & 0/0/0 & 0/0/0 & 0/0/0 & 7/1/0 & 0/0/0 & 0/0/0 & 0/0/0 & 0/0/0 & 0/0/0 & 0/0/0 & $3 / 0 / 0$ & $1 / 0 / 0$ & $0 / 0 / 0$ \\
\hline
\end{tabular}

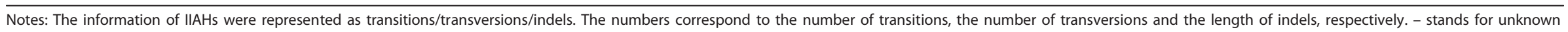
numbers due to the unavailiablity of sequences data 


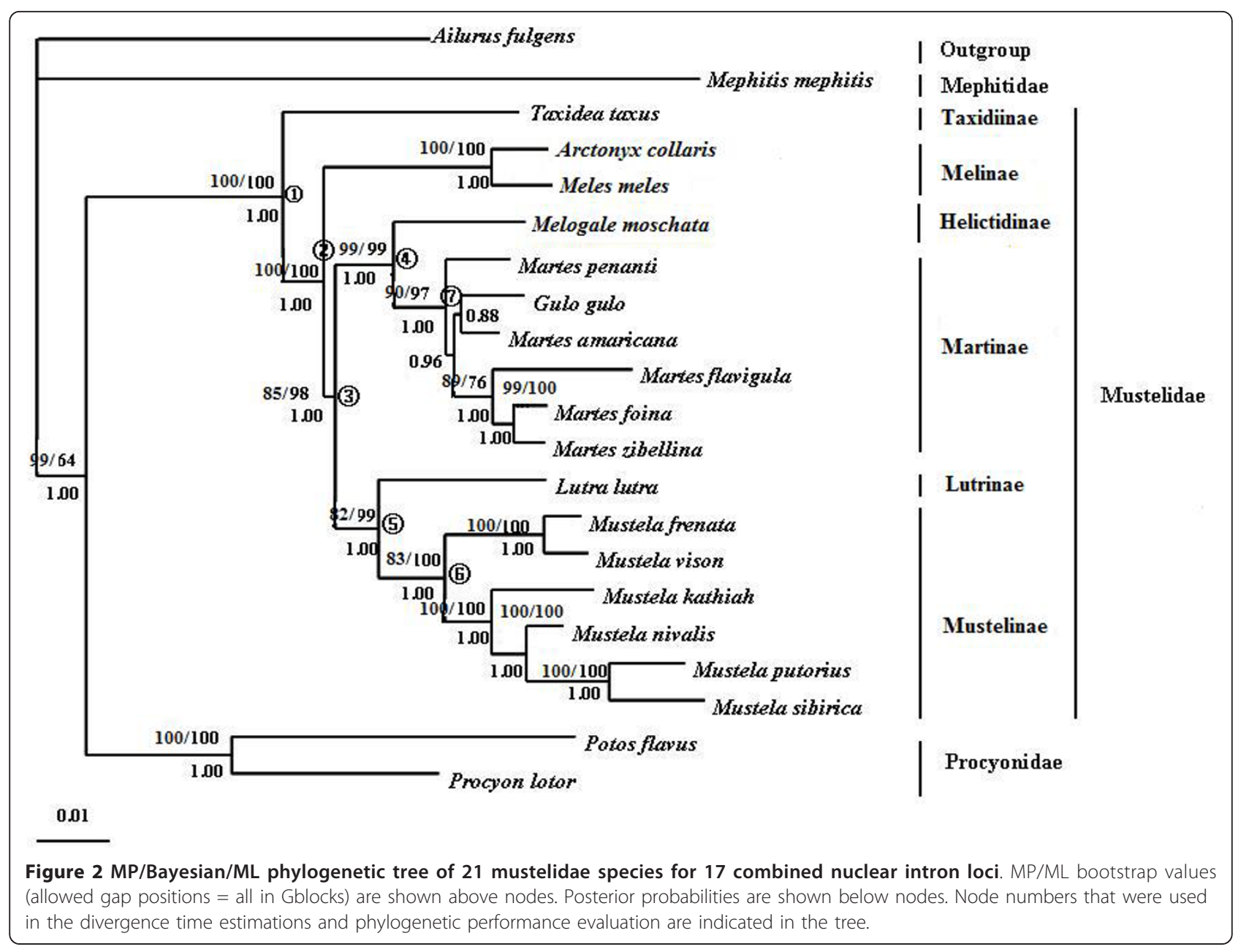

that, Melinae diverged from the other mustelids at 17.33 Mya (95\% confidence intervals $=12.44-22.10 \mathrm{Mya}$ ). The split between Martinae/Helictidinae and Lutrinae/Mustelinae took place at 16.05 Mya (95\% confidence intervals $=11.34-20.91 \mathrm{Mya})$. The divergence between Martinae and Helictidinae occurred at 10.21 Mya (95\% confidence intervals $=5.66-15.04 \mathrm{Mya})$, and that between Lutrinae and Mustelinae at 11.43 Mya (95\% confidence intervals $=7.02-16.00 \mathrm{Mya}$ ). The origins of Mustelinae and Martinae were estimated to be 5.89 Mya (95\% confidence intervals $=4.99-6.83 \mathrm{Mya})$ and 5.97 Mya (95\% confidence intervals $=3.39-8.90$ Mya), respectively.

The analyses of the mt genome data set suggested the origin of Taxidiinae around 23.46 Mya (95\% confidence intervals $=22.50-24.41 \mathrm{Mya})$. Other time estimates included the split of the Melinae clade from other mustelids at 17.19 Mya (95\% confidence intervals $=13.96$ 20.58 Mya), the separation between Helictidinae/Lutrinae/Mustelinae and Martinae about 15.79 Mya (95\% confidence intervals $=12.65-19.11 \mathrm{Mya})$, the divergence between Helictidinae and Lutrinae/Mustelinae at 13.86 Mya (95\% confidence intervals $=10.45-17.02 \mathrm{Mya}$ ), and the divergence between Lutrinae and Mustelinae at 12.54 Mya (95\% confidence intervals $=9.24-15.87 \mathrm{Mya}$ ). The origins of Mustelinae and Martinae were estimated to be $6.30 \mathrm{Mya}$ (95\% confidence intervals $=5.39-7.24$ Mya) and 10.91 Mya (95\% confidence intervals $=7.82$ $14.39 \mathrm{Mya})$, respectively.

\section{Discussion}

\section{Phylogeny of Mustelidae Subfamilies}

Among mammalian phylogenies, those characterized by rapid species radiations have long been one of the plaguing and challenging problems in species tree reconstruction [46]. This is the first study utilizing data from such large-scale nuclear non-coding loci from Mustelidae.

Both our combined nuclear intron and $\mathrm{mt}$ genome phylogenies not only strongly favor the prevailing view that Taxidiinae was the most basal member within family Mustelidae [4,5,10-14], but also provide strong 


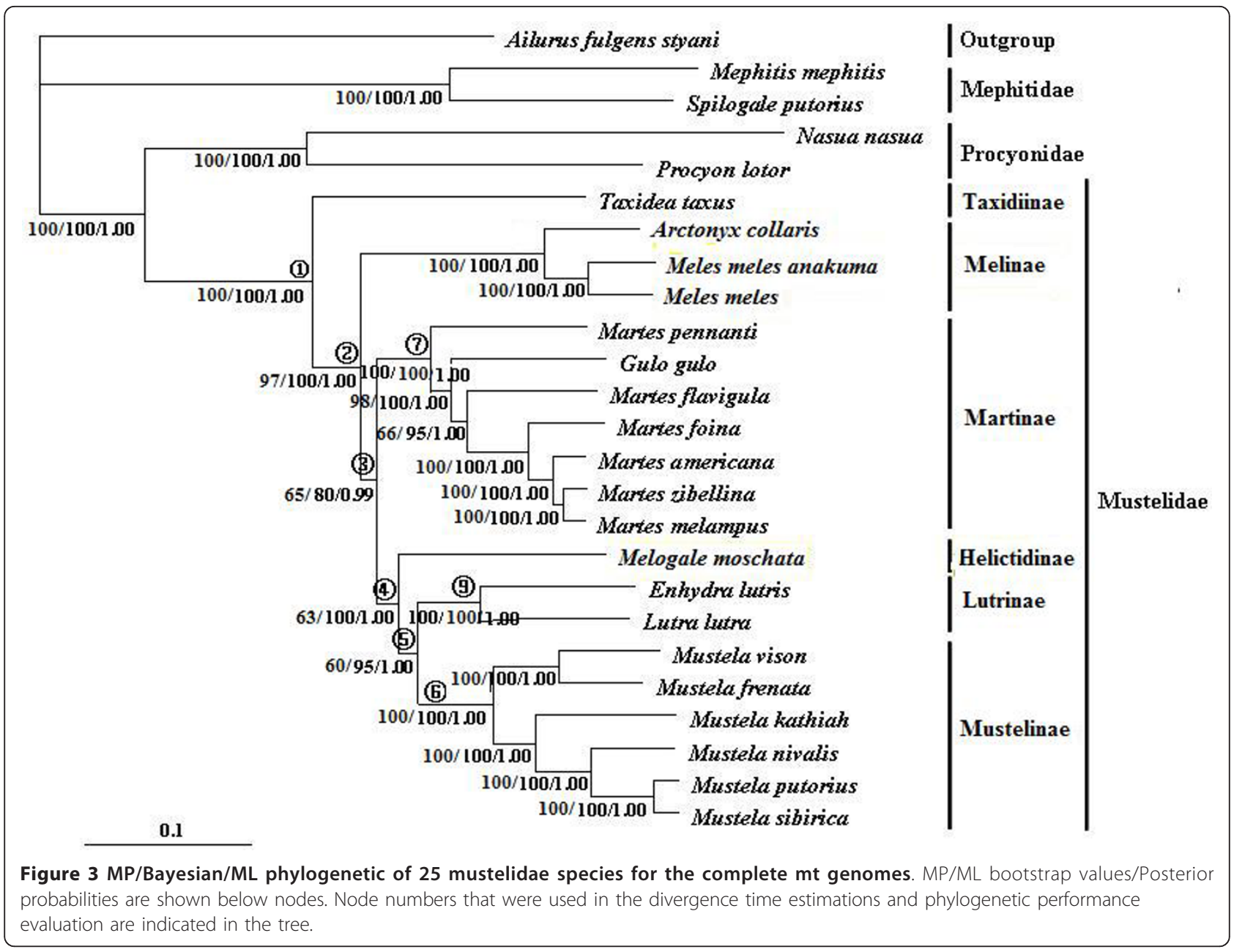

evidence that Melinae diverged between Taxidiinae and all the other mustelids examined as well. The latter is in contradiction to morphological investigations [8], but supports the nuclear gene results from Sato et al. $[11,12]$ and Wolsan and Sato [13], and disagree those from Koepfli et al. [5] and Yu et al. [15].

Notably, the sister relationship between Lutrinae and Mustelinae was reinforced by consistent recovery from both our mt genome and combined nuclear intron analyses with high confidence, upholding and strengthening the hypothesis drawn by almost all sequence-based analyses in previous studies $[4,5,7,10-15]$. In contrast, the position of Helictidinae varied between our nuclear and $\mathrm{mt}$ genome analyses. Nuclear data analysis placed it as sister to Martinae, whereas $\mathrm{mt}$ genome data indicated a sister-taxa association of it to the clade uniting Lutrinae and Mustelinae. Our mt genome result (Figure 3) is consistent with that inferred from most previous nuclear studies [3-5,10-13], but dissented from morphological view and karyological analyses [8,47-49]. Interestingly, our combined nuclear analysis (Figure 2) yields a result that is different from all previous hypotheses, suggesting for the first time Helictidinae and Martinae are more closely related to each other than any other taxa in Mustelidae.

Corresponding tests (AU and $\mathrm{KH}$ tests) have indicated significant topological incongruence between nuclear and $\mathrm{mt}$ trees. When using nuclear data, the $\mathrm{mt}$ genome tree topology was rejected by the AU and $\mathrm{SH}$ tests $(P<$ $0.05)$, and vice versa. Phylogenetic incongruence between nuclear and mitochondrial genes has also been reported in Drosophila, Aves and bears [50-53]. Various elements may bear the responsibility for the presence of conflicting signal regarding the placement of Helictidinae, including different evolutionary histories and gene properties in gene regions from different genomes, sampling error and lineage sorting. The probability of their occurrence increased especially when separation time between different species is short [54-57], as in the present study. Although the BCA analysis of the combined nuclear intron and $\mathrm{mt}$ genome sequences, which is an approach that allows for gene tree discordance, retrieved 


\begin{tabular}{|c|c|c|c|c|c|c|c|c|c|c|c|}
\hline Node & Diversification event & $\begin{array}{l}\text { Nuclear data }{ }^{a} \\
(95 \% \mathrm{CI})^{c}\end{array}$ & Mt $\operatorname{data}^{\mathrm{b}}(95 \% \mathrm{Cl})$ & Fossil record & $\begin{array}{l}\text { Wayne } \\
\text { (1989) }\end{array}$ & $\begin{array}{l}\text { Bininda- } \\
\text { Emonds } \\
\text { (1999) }\end{array}$ & $\begin{array}{l}\text { Hosoda } \\
\text { (2000) }\end{array}$ & Sato (2003) & $\begin{array}{l}\text { Yonezawa } \\
\text { (2007) }\end{array}$ & Koepli (2008) & $\begin{array}{l}\text { Eizirik et al } \\
\quad(2010)\end{array}$ \\
\hline 1 & $\begin{array}{l}\text { divergence of Taxidiinae to } \\
\text { the other mustelids }\end{array}$ & $23.73(22.80-24.70)$ & $23.46(22.50-24.41)$ & - & - & 20.8 & - & - & $20.21(18.78-21.64)$ & $24.2(22.3-26)$ & $13.0(9.6-17.1)$ \\
\hline 2 & divergence of Melinae & $17.33(12.44-22.10)$ & $17.19(13.96-20.58)$ & $18-20.5$ & - & 13.7 & - & $14.5-18.1$ & $16.98(15.52-18.44)$ & $11(9.4-12.5)$ & $11.7(8.5-15.5)$ \\
\hline 3 & $\begin{array}{l}\text { origin of the other } \\
\text { mustlids except for } \\
\text { Taxidiinae and Melinae }\end{array}$ & 16.05(11.34-20.91) & $15.79(12.65-19.11)$ & $21.5-22.5$ & - & 11.4 & - & $14.7-14.8$ & 14.83(13.39-16.27) & $11.6(10.1-13)$ & $10.8(7.8-14.5)$ \\
\hline 4 & divergence of Helictidinae & 10.21(5.66-15.04) & $13.86(10.45-17.02)$ & - & - & 6.9 & - & - & - & 10.8(9.4-12.2) & - \\
\hline 5 & $\begin{array}{l}\text { divergence between } \\
\text { Lutrinae and Mustelinae }\end{array}$ & $11.43(7.02-16.00)$ & $12.54(9.24-15.87)$ & $11.1-13.5$ & $20-25$ & $9.9-17.1$ & $15-23$ & $13.5-14.1$ & $12.74(11.41-14.07)$ & $8.7(7.3-10.0)$ & 8.4(5.9-11.5) \\
\hline 7 & origin of Mustelinae & $5.89(4.99-6.83)$ & 6.30(5.39-7.24) & $3.4-4.2$ & $12-20$ & $10.4-11.4$ & $10-23$ & $8.5-9.9$ & - & $6.1(4.9-7.2)$ & - \\
\hline 6 & origin of Martinae & 5.97(3.39-8.90) & $10.91(7.82-14.37)$ & $3.3-4.0$ & - & 8.2 & $10-14$ & - & $11.48(10.15-12.81)$ & $6.8(5.1-8.5)$ & $7.7(5.3-10.8)$ \\
\hline
\end{tabular}

Node numbers correspond to those indicated in Figure 2 and 3.

a the divergence times were estimated based on combined nuclear genes and tree topology in Figure 2 .

$\mathrm{b}$ the divergence times were estimated based on $\mathrm{mt}$ genomes and tree topology in Figure 3 .

c $95 \%$ confidence intervals. 
Table 5 Phylogenetic performance of nuclear and $\mathrm{mt}$ genes

\begin{tabular}{|c|c|c|c|c|c|c|c|c|c|c|c|c|c|}
\hline \multicolumn{2}{|c|}{ Gene $^{a}$} & \multirow{2}{*}{$\begin{array}{c}\text { no. congruent branches (BP > } \\
0.95)\end{array}$} & \multirow{2}{*}{$\begin{array}{c}\text { no. congruent branches }(\mathrm{BP}< \\
0.95)\end{array}$} & \multirow{2}{*}{$\begin{array}{l}\text { total no. congruent } \\
\text { branches }\end{array}$} & \multicolumn{8}{|c|}{ Node $^{b}$} & \\
\hline & & & & & 1 & 2 & 3 & 4 & 5 & 6 & 7 & 8 & $\overline{9}$ \\
\hline \multirow[t]{16}{*}{ Mt gene } & nd5 & 8 & 1 & 9 & * & * & * & $\#$ & * & * & * & $*$ & * \\
\hline & cytb & 8 & 1 & 9 & * & ${ }^{*}$ & * & * & \# & * & * & * & * \\
\hline & 16sRNA & 8 & 0 & 8 & * & * & & * & * & $*$ & * & $*$ & $*$ \\
\hline & $\mathrm{nd} 2$ & 6 & 1 & 7 & * & & \# & * & & * & * & * & * \\
\hline & $\cos 2$ & 6 & 0 & 6 & * & & * & * & & * & * & * & \\
\hline & nd4 & 6 & 0 & 6 & * & & & & * & * & * & * & * \\
\hline & nd6 & 5 & 1 & 6 & * & * & & & & * & * & * & $\#$ \\
\hline & 12sRNA & 5 & 1 & 6 & * & $\#$ & & & & * & * & * & * \\
\hline & nd3 & 4 & 2 & 6 & * & $\#$ & & & & * & $\#$ & * & * \\
\hline & $\operatorname{cox} 1$ & 5 & 0 & 5 & * & & & & & * & * & * & $*$ \\
\hline & $\operatorname{cox} 3$ & 5 & 0 & 5 & * & * & & \# & & * & * & * & \\
\hline & tRNAs & 4 & 1 & 5 & * & $\#$ & & & & & * & $*$ & $*$ \\
\hline & nd1 & 4 & 1 & 5 & * & & & & & $\#$ & * & * & * \\
\hline & atp8 & 3 & 1 & 4 & * & $\#$ & & & & $*$ & & * & \\
\hline & atp6 & 4 & 0 & 4 & * & & & & & * & & * & * \\
\hline & nd4l & 2 & 0 & 2 & & & & & & * & \# & * & \\
\hline \multirow[t]{17}{*}{$\begin{array}{l}\text { Nuclear } \\
\text { gene }\end{array}$} & $\begin{array}{l}\text { Plod2- } \\
13\end{array}$ & 6 & 2 & 8 & * & * & $\#$ & \# & * & * & * & * & \\
\hline & $\begin{array}{l}\text { Plod2- } \\
14\end{array}$ & 6 & 1 & 7 & * & * & * & * & \# & * & & * & \\
\hline & $\begin{array}{l}\text { Corolc- } \\
5\end{array}$ & 5 & 1 & 6 & * & $*$ & & * & \# & * & - & * & \\
\hline & $\begin{array}{l}\text { Tinagl1- } \\
3\end{array}$ & 5 & 1 & 6 & * & * & \# & * & - & * & & * & \\
\hline & $\begin{array}{l}\text { Corolc- } \\
4\end{array}$ & 6 & 0 & 6 & * & & * & & * & * & * & * & \\
\hline & $\begin{array}{l}\text { Tinagl1- } \\
1\end{array}$ & 6 & 0 & 6 & * & $*$ & & * & * & * & & * & \\
\hline & Cidea-1 & 6 & 0 & 6 & * & $*$ & * & * & - & * & & * & \\
\hline & $\begin{array}{l}\text { Ociad1- } \\
4\end{array}$ & 4 & 2 & 6 & * & $\#$ & * & * & \# & & & * & \\
\hline & Wasf1-3 & 5 & 1 & 6 & * & $*$ & & * & \# & * & & * & \\
\hline & Fgb-7 & 5 & 1 & 6 & \# & * & & * & * & * & & * & \\
\hline & Ttr-1 & 6 & 0 & 6 & * & * & & * & * & * & - & * & \\
\hline & Wasf1-6 & 5 & 0 & 5 & * & $*$ & & * & * & & & * & \\
\hline & Fgb-4 & 4 & 1 & 5 & * & & & * & \# & * & & * & \\
\hline & Wasf1-7 & 3 & 0 & 3 & $*$ & * & & & & & & * & \\
\hline & $\begin{array}{l}\text { Guca1b- } \\
3\end{array}$ & 2 & 0 & 2 & * & & & & & & & * & \\
\hline & Ssr1-5 & 2 & 0 & 2 & * & & & & & & & * & \\
\hline & $\begin{array}{l}\text { Tbc1d7- } \\
6\end{array}$ & 0 & 0 & 0 & & & & & & & & & \\
\hline
\end{tabular}

Node numbers correspond to those indicated in Figure 2 and 3.

a genes are ranked by the total number of congruent branches in the combined topologies.

$\mathrm{b}$ there are 9 nodes in total indicated in the $\mathrm{mt}$ genome tree (Figure 3) and 8 nodes in the combined nuclear gene tree (Figure 2).

* branches with PP $>0.95$ congruent in the combined topology.

\# branches with PP $<0.95$ congruent in the combined topology. 
an identical tree topology to that of the nuclear intron gene, the position of Helictidinae received week support. Therefore, the analyses involving more characters in the future may help to confirm the precise position of Helictidinae.

\section{Implications for Mustelidae Radiation}

Mustelidae has one of the most extensive fossil records of extant Carnivora families [8], and molecular dating of the Mustelidae radiation has also been attempted in several studies previously. Our results, from an independent character source, provide important insights into the time scale of the origin and diversification of extant subfamilies of Mustelidae. It is interesting to draw a comparison of the dating results estimated from prior and present studies (Table 4).

Among the subfamilies, Taxidiinae is the basal-most branching lineage in mustelid diversification dating to the late Oligocene, which is more concordant with the time estimate of Koepfli et al. [5], but earlier than those of Bininda-Emonds et al. [58], Yonezawa et al. [15] and Eizirik et al. [59]. The divergence time of the next branching lineage, i.e., Melinae clade, is dated to the early Miocene in our analysis, which is more broadly consistent with the paleontological data [60] and the sequence-based data from Yonezawa et al. [15] than those from Sato et al. [2], Bininda-Emonds et al. [54], Koepfli et al. [5], and Eizirik et al [59]. The estimates of the origin of the remaining mustelids are more recent than those fossil-based ages [61], and older than the other sequence-based dates $[2,5,15,58,59]$.

Among the remaining mustelids, one important event of the mustelid diversification is the divergence between Lutrinae and Mustelinae. Our estimated dates are consistent with the corresponding fossil records $[60,62]$ and that from Yonezawa et al. [15], but more recent than those from Hosoda et al. [63], Wayne [64], and Sato et al. [2], and older than those from Koepfli et al. [5] and Eizirik et al. [59]. Other important events are the origins of Mustelinae and Martinae. Our estimates for them are both much older than the existing fossil record $[65,66]$. The dates of Mustelinae origin are older than most of the other molecular estimates, but in good agreement with that of Koepfli et al. [5]. As regards the origin of Martinae, there is large difference between our nuclear and $\mathrm{mt}$ genome estimates. The nuclear estimate is more in agreement with those from Koepfli et al. [5] and Eizirik et al. [59], while the mt estimate is more concordant with those from Hosoda et al. [63] and Yonezawa et al. [15].

In addition, our analyses resulted in time estimates of divergence of Helictidinae that more agree with that from Koepfli et al. [5] than that from Bininda-Emonds et al. [58], which is younger than the present results.
More intensive taxonomic sampling will improve accuracy on the time estimation of mustelid diversification.

\section{Utilities of the nuclear introns in phylogenetic study of Mustelidae subfamilies}

Several recent studies have indicated that nuclear introns hold considerable signals for resolution of difficult phylogenies at both shallow and deeper species level hierarchies $[21-24,27,67,68]$. We are among the first to use large-scale nuclear intron genes in inferring phylogenies of Mustelidae. Our analysis not only brings new perspectives on the phylogenetic relationship of Mustelidae subfamilies, but provides another example demonstrating that the nuclear non-coding genes can be an effective data source for reconstructing evolutionary histories in a group that has undergone rapid bursts of speciation as well.

We assessed the phylogenetic utilities of individual introns and $\mathrm{mt}$ genes in resolution of the inter-subfamilial relationships of Mustelidae by counting the number of congruent nodes between the individual phylogenies and the combined gene trees (Table 5). In the individual nuclear gene analyses, the Plod2-13 gene recovered all 8 nodes of the combined nuclear gene tree. Anyway, the Plod2-13 and Plod2-14 genes recovered the highest number of congruent nodes of the combined nuclear gene tree, whereas the Wasf1-7, Guca1b-3, Ssr1-5, and Tbc1d7-6 genes showed the lowest phylogenetic performance. As regards the mt gene analyses, we observed that the ND5 and CYTB genes recovered all 9 nodes of the $\mathrm{mt}$ genome tree. Ranking the single $\mathrm{mt}$ gene shows that the ND5, CYTB, 16SrRNA and ND2 genes are better indicators of Mustelidae phylogeny at subfamilial level than are other genes, such as Atp 8, Atp 6 and $N D 4 L$ genes. This result agrees broadly with previous conclusions about the rough classification of $\mathrm{mt}$ genes into good, medium, and poor performance categories [69-74] (Additional file 4). In summary, the assessment of phylogenetic utility and limits of these individual nuclear and mt genes makes it possible to preselect subsets of genes for future molecular studies of vertebrate phylogeny.

Although the use of nuclear introns as genetic markers has now been implemented as a powerful approach in recent phylogenetic studies, there are, however, numerous potential problems associated with this approach. Chief among these is the difficulties in sequence data acquisition, alignment, and analysis of the nuclear introns compared to the traditional $\mathrm{mt}$ and nuclear protein-coding genes, as a result of the higher rates of variation and frequencies of indels. The common presence of indels (including TEs, small gaps and tandem repeats) and IIAHs, as reported in this study, makes experimental work labor-intensive by virtue of 
the additional time and money required to isolate alleles and optimize PCR amplification and sequencing. In addition, they can create positional homology problems associated with areas of ambiguous alignment [75]. Several studies have also shown that the phylogenetic inference is sensitive to the various treatments of indels. These issues are central to the appropriate application of intron data in phylogenetic reconstruction and they should be comprehensively and explicitly addressed in the future studies [19].

\section{Conclusions}

The phylogenetic relationships among Mustelidae subfamilies have posed one of the major problems concerning Carnivora systematics. In this study, phylogenetic relationships among Mustelidae subfamilies are presented based on 17 nuclear intron loci and mt whole genomes. Our results resolve some of the ambiguous issues in Mustelidae phylogeny, whereas some phylogenetic relationships require confirmation by analyzing additional samples and character information, such as the precise position of Helictidinae. Our study not only brings new perspectives on the previously obscured phylogenetic relationships among Mustelidae subfamilies, but also provides another example demonstrating the effectiveness of nuclear non-coding loci for reconstructing evolutionary histories in a group that has undergone rapid bursts of speciation.

\section{Methods}

\section{Sequence Data}

Detailed information of the 14 nuclear intron loci first used in the Mustelidae phylogeny is shown in Additional file 5. These loci were amplified with primers as described in Yu et al. [27] from 17 mustelids and 4 nonmustelid carnivoran species. Three other nuclear introns (Ttr-1, Fgb-4 and Fgb-7), which were available from our previous published studies [14,76,77], were also included in the present nuclear data set.

The species examined in this study and their Genbank accession numbers are listed in Additional file 6. A "touch-down" PCR amplification was carried out using the following parameters: $95^{\circ} \mathrm{C}$ hot start $(5 \mathrm{~min}), 10$ cycles of $94^{\circ} \mathrm{C}$ denaturation $(1 \mathrm{~min}), 60-50^{\circ} \mathrm{C}$ annealing $(1 \mathrm{~min}), 72^{\circ} \mathrm{C}$ extension $(1 \mathrm{~min})$, and finally 25 cycles of $94^{\circ} \mathrm{C}$ denaturation $(1 \mathrm{~min}), 50^{\circ} \mathrm{C}$ annealing $(1 \mathrm{~min}), 72^{\circ} \mathrm{C}$ extension $(1 \mathrm{~min})$. The amplified DNA fragments were purified and sequenced in both directions with an ABI PRISM(tm) 3730 DNA sequencer following the manufacturer's protocol. In the case of poor performance of direct sequencing resulting from complex DNA structures, tandem repeats or intron heterozygotes, the amplified PCR products were gel-purified and cloned into the pMD18-T vector (TaKaRa Biotechnology Co.,
Ltd. Dalian, China) and transformed into ultracompetent E. coli cells (TaKaRa Biotechnology Co., Ltd. Dalian, China). Thirty positive clones per ligation reaction were sequenced. All sequences obtained were checked carefully and queried in BLAST searches of GenBank to assess homology. In a few cases, PCR attempts using different primer pairs and cloning methods failed to produce sequence data (see Additional file 6). These sequences were excluded from the independent gene analyses and treated as missing data in the combined analyses. The newly determined nuclear sequences have been deposited in GenBank with accession numbers HM063147-HM063412.

The mt complete genome sequences were amplified using LA PCR ${ }^{\mathrm{TM}}$ Kit (Takara Biotechnology Co., Ltd) and 9 universal long PCR primers from 14 mustelids and 2 non-mustelid carnivoran species (Additional file 6). In addition, 23 species-specific primers were designed when the universal PCR primers failed to produce successful PCR amplification. A "touch-down" long PCR amplification was carried out using the following parameters: $95^{\circ} \mathrm{C}$ hot start $(2 \mathrm{~min}), 10$ cycles of $98^{\circ} \mathrm{C}$ denaturation $(10 \mathrm{sec}), 67-58^{\circ} \mathrm{C}$ annealing $(1 \mathrm{~min} 30 \mathrm{sec}$; ${ }^{\circ} \mathrm{C} /$ cycle), $72^{\circ} \mathrm{C}$ extension $(5 \mathrm{~min})$, and finally 25 cycles of $98^{\circ} \mathrm{C}$ denaturation $(10 \mathrm{sec}), 58^{\circ} \mathrm{C}$ annealing $(1 \mathrm{~min} 30$ sec), $72^{\circ} \mathrm{C}$ extension (5 min). At the end, a final 10 -min extension at $72^{\circ} \mathrm{C}$ was performed. Long PCR products were sequenced in both directions using a primer walking strategy with a total of 306 primers. Sequencing was performed in an ABI PRISM(tm) 3700 DNA sequencer following the manufacturer's protocol. Primer sequence information is available upon request. Where necessary, PCR products were cloned into the pMD18-T vector and transformed into ultracompetent E. coli cells (TaKaRa Biotechnology Co., Ltd. Dalian, China) in order to resolve the difficulty of direct sequencing of control regions arising from long tandem repeats. Five positive clones per ligation reaction were sequenced. Mt sequences obtained were checked to ensure that they did not include nuclear copies of mtDNA-like pseudogenes. In addition to these new $16 \mathrm{mt}$ genomes, 6 other mustelid and 3 other non-mustelid mt genomes available in public database were included in the $\mathrm{mt}$ analyses (Additional file 6).

\section{Alignments and sequence Characterizations}

Sequences were aligned using CLUSTAL X under the default settings [78]. The nuclear alignment was divided into two data sets: (1) each of the 17 intron loci and (2) combined sequences of all introns. The mt alignment was divided into four data sets: (1) each of the 13 protein-coding genes, (2) 22 tRNAs, (3) two rRNAs, and (4) combined sequences of the tRNAs, rRNAs and proteincoding genes. Due to the presence of ambiguous areas 
in the nuclear alignments, we excluded the alignment ambiguities using Gblocks 0.91b [79] with default parameters (allowed gap positions $=$ all).

Pairwise comparisons and sequence characterizations were estimated using MEGA 4.0 [80]. Given that nuclear introns tend to be favorable chromosomal regions for integration of transposable elements (TEs) [81], they were screened for interspersed repeats by using the program RepeatMasker (Smit, Hubley and Green, RepeatMasker Open-3.0. 1996-2004, http://www. repeatmasker.org).

\section{Phylogenetic Analyses}

Phylogenetic analyses of the individual introns and $\mathrm{mt}$ genes, i.e., nuclear data set (1) and $\mathrm{mt}$ data set (1), (2), and (3), were performed using PAUP* 4.0b10 [82] for maximum parsimony (MP) and maximum likelihood (ML) analyses, and using MrBayes 3.1.2 [83] for the Bayesian inference. In MP analyses, a heuristic search was performed with tree-bisection-reconnection (TBR) branch swapping, random addition of taxa, and 1000 replicates per search. Only one of the best trees found during branch swapping was saved. In ML analysis, the best-fit models of sequence evolution were selected using the Akaike Information Criterion (AIC) $[84,85]$ with Modeltest version 3.7 [86]. The chosen models and their parameters were used to infer ML trees with the heuristic algorithm, 10 random-addition sequence replicates, and TBR branch swapping. The tree reliability under ML analysis was assessed using a bootstrap resampling of 100 replicates (BP) [87]. In Bayesian inference, each Metropolis-coupled Markov chain Monte Carlo (MCMC) run for all individual genes employed the model selected by ModelTest for that gene, or the nearest model to that model that could be implemented in MrBayes. Three heated chains and a single cold chain were used in all MCMC analyses and run for $2 \times 10^{6}$ generations, Trees were sampled every 100 generation. The average standard deviation of split frequencies was close to 0.001 when the run was end. The first $25 \%$ were discarded as the burn-in. A 50\% majority-rule consensus of post burn-in trees was constructed to summarize posterior probabilities (PP) for each branch.

In addition to individual analyses, phylogenetic reconstruction were performed based on the combined data sets, i.e., nuclear data set (2) and mt data set (4), using PAUP* 4.0b10 [82] for MP analysis, RAxML online web server [88] for partitioned ML analysis and using MrBayes [83] for partitioned Bayesian analysis (pBI) [89]. For the combined data sets, we identified model partitions based on partitioning matrices by locus. That is, in the analysis of combined nuclear data set, each nuclear intron gene was considered as a different partition, whereas in that of combined mt data set, each of the 13 individual protein-coding genes, all tRNAs, and each of the two rRNA genes were considered as different partitions. Based on the selected models using the AIC [84] as mentioned above for individual analyses, we assigned a separate substitution model for each of the data partitions. Three heated chains and a single cold chain were used in all MCMC analyses and run for $5 \times$ $10^{6}$ generations, sampling trees every 100 generations. The average standard deviation of split frequencies was close to 0.001 when the run was end. The first $25 \%$ were discarded as the burn-in. A 50\% majority-rule consensus of post burn-in trees was constructed to summarize posterior probabilities (PP) for each branch.

In addition, given the heterogeneous gene trees observed among 17 nuclear intron gene analyses and between the combined nuclear intron and $\mathrm{mt}$ genome analyses, Bayesian concordance analysis (BCA) [45] implemented in the program BUCKy [45], which uses individually calculated gene trees to infer the species tree that maximizes the bipartition concordance among each gene tree, was also performed for both the nuclear intron gene datasets and the nuclear plus the mt genome datasets. Tree reliability was evaluated by sample concordance factors (CFs). The MCMCMC sampled with $2 \times 10^{6}$ generations was employed (4 runs and 4 chains) and a priori level of discordance $\alpha=2.5$ was used in BCA.

In all analyses, trees were rooted with the red panda Ailurus fulgens and the skunk Mephitis mephitis, based on the general consensus that they branched off earlier than the mustelids $[2,4,11,12,76,77]$.

\section{Intra-individual allele heterozygotes}

For individual intron analyses, both copies of alleles from a species were included. For combined nuclear data sets, we performed phylogenetic analyses by (1) choosing randomly an allele per species for portioned $\mathrm{ML}$ analysis and Bayesian analysis (without the inclusion of IIAHs), and (2) using POFAD v1.03 algorithm (Phylogeny Analysis From Allelic Data) [90] to incorporate IIAHs. POFAD is a recently developed method of constructing phylogeny from multiple datasets that contain allelic information. It converts a distance matrix of alleles into a distance matrix of organisms so that individuals become the terminals of the analyses. First, we calculated the average uncorrected pairwise distances in PAUP* [82]. These distances then served as the input for the calculation of standardized pairwise distances between species in POFAD [90]. The standardized distances were then used as input for the neighbor-joining analysis conducted using PAUP* [82] to produce a phylogenetic tree. 


\section{Divergence time estimation}

Divergence times based on combined nuclear intron and combined $\mathrm{mt}$ data sets were estimated using the Bayesian relaxed phylogenetic approach implemented in BEAST v1.5.4 [91]. We assumed a GTR $+\mathrm{I}+\mathrm{G}$ model of DNA substitution with four rate categories. Uniform priors were employed for GTR substitution parameters $(0,100)$, gamma shape parameter $(0,100)$ and proportion of invariant sites parameter $(0,1)$. The uncorrelated lognormal relaxed molecular clock model was used to estimate substitution rates for all nodes in the tree, with uniform priors on the mean $(0,100)$ and standard deviation $(0$, $10)$ of this clock model. We employed the Yule process of speciation as the tree prior and a UPGMA tree to construct a starting tree, with the ingroup assumed to be monophyletic with respect to the outgroup.

Three calibration points from the fossil records were applied in the dating analyses. These calibration points are all implemented as minimum age constraints, including 27.6 Mya for the split between Procyonidae and Mustelidae [12,15,92], 24 Mya for the crown Mustelidae [2,5,17], and 5.3 Mya for the origin of the genus Mustela in Mustelinae [5,93]. All fossil constraint priors were set as means of a normal distribution, with a standard deviation of 1.0 MYA. Two independent MCMC runs of 30,000,000 generations were performed for each data set with parameters logged every 1,000 generations. The Auto Optimize Operators function was enabled to maximize efficiency of MCMC runs. Two independent MCMC runs for each analysis were combined to estimate the posterior distribution of the substitution model and tree model parameters, as well as node ages. Analyses of these parameters in Tracer 1.5 [94] suggested that the number of MCMC steps was more than adequate, with effective sample sizes of all parameters often exceeding 1,000 and Tracer plots showing strong equilibrium after discarding burn-in.

\section{Testing Tree Incongruence}

The incongruence among different tree topologies was evaluated using the Shimodaira-Hasegawa (SH) test [95] and the approximately unbiased (AU) test [96], as implemented in the CONSELV0.1i program [97] with default scaling and replicate values. The site-wise loglikelihood values were estimated by PAUP* [82].

\section{Additional material}

Additional file 1: Phylogenetic relationships of Mustelidae based on the analyses of 17 single introns. The IIAHs within a species was shown as 1 and 2. All trees shown were reconstructed using Bayesian method. Posterior probabilities (PP) are shown above internal nodes.

Additional file 2: Phylogenetic relationships of Mustelidae based on the Bayesian concordance analysis (BCA) of the nuclear intron gene datasets and the nuclear plus the $\mathrm{mt}$ genome datasets. The concordance factors (CFs) from the nuclear intron gene analysis and the nuclear plus the mt genome analysis are shown above internal nodes.

Additional file 3: Phylogenetic relationships of Mustelidae based on the analyses of 13 individual protein-coding genes, 2 individual rRNA genes, 22 tRNAs, combined protein-coding genes, combined rRNA genes, and combined tRNA genes. All trees shown were reconstructed using Bayesian method. Posterior probabilities (PP) are shown above internal nodes.

Additional file 4: Comparisons of phylogenetic performances of $\mathrm{mt}$ genes among studies.

Additional file 5: Detailed information of 14 nuclear intron loci that were first used in the Mustelidae phylogeny.

Additional file 6: The species examined in this study and their Genbank accession numbers.

\section{Acknowledgements}

We would like to give thanks to Mammalogy Collections of the Department of Natural History, Royal Ontario Museum, for providing some samples. This work was supported by grants from the State Key Basic Research and Development Plan (2007CB411600), and National Natural Science Foundation of China (30600067, U083660). We thank C. Steiner for helpful comments on the manuscript.

\section{Author details}

'Laboratory for Conservation and Utilization of Bio-resource \& Key Laboratory for Microbial Resources of the Ministry of Education, Yunnan University, Kunming, 650091, PR, China. ${ }^{2}$ State Key Laboratory of Genetic Resources and Evolution, Kunming Institute of Zoology, Kunming 650223, China. ${ }^{3}$ Conservation Genome Resource Bank for Korean Wildlife, Research Institute for Veterinary Science and Coll. of Vet. Med., Seoul National Univ., Seoul 151-742, South Korea. ${ }^{4}$ San Diego Zoo's Institute for Conservation Research, Escondido, CA 92027-7000, USA.

\section{Authors' contributions}

$L Y, Y P Z$ and OAR designed the study and wrote the manuscript. DP and JL carried out the experiment work and performed the sequence analyses. PTL and $\mathrm{LL}$ contributed to the experiment work and sequence analyses. $\mathrm{HL}$ and ML helped collect samples. All authors read and approved the final manuscript.

Received: 2 January 2011 Accepted: 10 April 2011

Published: 10 April 2011

\section{References}

1. Nowak RM: Walker's mammals of the world. Baltimore, MaryLand: Johns Hopkins University Press; 61999.

2. Sato JJ, Hosoda T, Wolsan M, Tsuchiya K, Yamamoto Y, Suzuki H: Phylogenetic relationships and divergence times among mustelids (Mammalia: Carnivora) based on nucleotide sequences of the nuclear interphotoreceptor retinoid binding protein and mitochondrial cytochrome b genes. Zool Sci 2003, 20:243-264.

3. Sato JJ, Hosoda T, Wolsan M, Suzuki H: Molecular phylogeny of arctoids (Mammalia: Carnivora) with emphasis on phylogenetic and taxonomic positions of ferret-badgers and skunks. Zool Sci 2004, 21:111-118.

4. Fulton TL, Strobeck C: Molecular phylogeny of the Arctoidea (Carnivora): effect of missing data on supertree and supermatrix analyses of multiple gene data sets. Mol Phylogenet Evol 2006, 41:165-181.

5. Koepfli KP, Deere KA, Slater GJ, Begg C, Begg K, Grassman L, Lucherini M, Veron G, Wayne RK: Multigene phylogeny of the Mustelidae: Resolving relationships, tempo and biogeographic history of a mammalian adaptive radiation. BMC Biol 2008, 6:10.

6. Koepfli KP, Wayne RK: Phylogenetic relationships of otters (Carnivora; Mustelidae) based on mitochondrial cytochrome b sequences. J Zool 1998, 246:401-416.

7. Marmi J, López-Giráldez J, Domingo-Roura X: Phylogeny, evolutionary history and taxonomy of the Mustelidae based on sequences of the cytochrome b gene and a complex repetitive flanking region. Zool Scri 2004, 33:481-499. 
8. Bryant HN, Russell AP, Fitch WB: Phylogenetic relationships within the extant Mustelidae (Carnivora): appraisal of the cladistic status of the Simpsonian subfamilies. Zool J Linn Soc 1993, 108:301-334.

9. Dragoo JW, Honeycutt RL: Systematics of mustelid-like carnivores. J Mamm 1997, 78:426-443.

10. Koepfli KP, Wayne RK: Type 1 STS markers are more informative than cytochrome $\mathrm{b}$ in phylogenetic reconstruction of the Mustelidae (Mammalia: Carnivora). Syst Biol 2003, 52:571-593.

11. Sato JJ, Wolsan M, Suzuki H, Hosoda T, Yamaguchi Y, Hiyama K Kobayashi M, Minami S: Evidence from nuclear DNA sequences sheds light on the phylogenetic relationships of Pinnipedia: single origin with affinity to Musteloidea. Zool Sci 2006, 23:125-146.

12. Sato JJ, Wolsan M, Minami S, Hosoda T, Sinaga MH, Hiyama K, Yamaguchi $Y$, Suzuki H: Deciphering and dating the red panda's ancestry and early adaptive radiation of Musteloidea. Mol Phylogenet Evol 2009, 53:907-922.

13. Wolsana M, Sato JJ: Effects of data incompleteness on the relative performance of parsimony and Bayesian approaches in a supermatrix phylogenetic reconstruction of Mustelidae and Procyonidae (Carnivora). Cladistics 2010, 26:168-194.

14. Yu L, Liu J, Luan PT, Lee H, Lee M, Min MS, Ryder OA, Chemnick L, Davis H, Zhang YP: New insights into the evolution of intronic sequences of the beta-fibrinogen gene and their application in reconstructing mustelid phylogeny. Zool Sci 2008, 25:662-672.

15. Yonezawa T, Nikaido M, Kohno N, Fukumoto Y, Okada N, Hasegawa M: Molecular phylogenetic study on the origin and evolution of Mustelidae. Gene 2007, 396:1-12.

16. Wolsan M: Phylogeny and classification of early European Mustelida (Mammalia: Carnivora). Acta Theriol 1993, 38:345-384.

17. Wolsan M: Oldest mephitine cranium and its implications for the origin of skunks. Acta Palaeontol Pol 1999, 44:223-230.

18. Wolsan M: Fossil-based minimum divergence dates for the major clades of musteloid carnivorans. Abstracts of Plenary, Symposium, Poster and Oral Papers Presented at Ninth International Mammalogical Congress (IMC 9) 2005, 372-373.

19. Creer S, Pook CE, Malhotra A, Thorpe RS: Optimal intron analyses in the trimeresurus radiation of Asian pitvipers. Syst Biol 2006, 55:57-72.

20. Beltrán $M$, Jiggins $C D$, Bull V, Linares $M$, Mallet J, McMillan WO, Bermingham E: Phylogenetic discordance at the species boundary: comparative gene genealogies among rapidly radiating Heliconius butterflies. Mol Biol Evol 2002, 19:2176-2190.

21. Matthee CA, Eick G, Willows-Munro S, Montgelard C, Pardini AT, Robinson TJ: Indel evolution of mammalian introns and the utility of non-coding nuclear markers in eutherian phylogenetics. Mol Phylogenet Evol 2007, 42:827-837.

22. Möller-Krull M, Delsuc F, Churakov G, Marker C, Superina M, Brosius J, Douzery EJ, Schmitz J: Retroposed elements and their flanking regions resolve the evolutionary history of xenarthran mammals (armadillos, anteaters, and sloths). Mol Biol Evol 2007, 24:2573-2582.

23. Dalebout ML, Steel D, Baker CS: Phylogeny of the beaked whale genus Mesoplodon (Ziphiidae: Cetacea) revealed by nuclear introns: implications for the evolution of male tusks. Syst Biol 2008, 57:857-875

24. Schröder C, Bleidorn C, Hartmann S, Tiedemann R: Occurrence of CanSINEs and intron sequence evolution supports robust phylogeny of pinniped carnivores and their terrestrial relatives. Gene 2009, 448:221-226.

25. Friesen V: Introns. In Molecular Methods in Ecology. Edited by: Baker AJ. Oxford: Blackwell Science Ltd; 2000:274-294.

26. Friesen $\mathrm{VL}$, Congdon $\mathrm{BC}$, Walsh $\mathrm{HE}$, Birt TP: Intron variation in marbled murrelets detected using analyses of single-stranded conformational polymorphisms. Mol Ecol 1997, 6:1047-1058.

27. Yu L, Luan PT, Jin W, Ryder OA, Chemnick LG, Davis HA, Zhang YP: Phylogenetic Utility of Nuclear Introns in Interfamilial Relationships of Caniformia (Order Carnivora). Syst Biol 2011, 60:175-187.

28. Jurka J, Zietkiewicz E, Labuda D: Ubiquitous mammalian-wide interspersed repeats (MIRs) are molecular fossils from the mesozoic era. Nucleic Acids Res 1995, 23:170-175.

29. Smit AF, Riggs AD: MIRs are classic, tRNA-derived SINEs that amplified before the mammalian radiation. Nucleic Acids Res 1995, 23:98-102.

30. Waterston RH, Lander ES, Sulston JE: On the sequencing of the human genome. Proc Natl Acad Sci USA 2002, 99:3712-3716.

31. Minnick MF, Stillwell LC, Heineman JM, Stiegler GL: A highly repetitive DNA sequence possibly unique to canids. Gene 1992, 110:235-238.
32. Coltman DW, Wright JM: Can SINEs: a family of tRNA-derived retroposons specific to the superfamily Canoidea. Nucleic Acids Res 1994, 22:2726-2730

33. van der Vlugt HH, Lenstra JA: SINE elements of carnivores. Mamm Genome 1995, 6:49-51.

34. Das M, Chu LL, Ghahremani M, Abrams-Ogg T, Roy MS, Housman D, Pelletier J: Characterization of an abundant short interspersed nuclear element (SINE) present in Canis familiaris. Mamm Genome 1998, 9:64-69.

35. Pecon-Slattery J, Pearks Wilkerson AJ, Murphy WJ, O'Brien SJ: Phylogenetic assessment of introns and SINEs within the $Y$ chromosome using the cat family felidae as a species tree. Mol Biol Evol 2004, 21:2299-2309.

36. Zehr SM, Nedbal MA, Flynn JJ: Tempo and mode of evolution in an orthologous Can SINE. Mamm Genome 2001, 12:38-44.

37. Vassetzky NS, Kramerov DA: CAN-a pan-carnivore SINE family. Mamm Genome 2002, 13:50-57.

38. Yu L, Zhang YP: Evolutionary implications of multiple SINE insertions in an intronic region from diverse mammals. Mamm Genome 2005, 16:651-660.

39. Nikaido M, Matsuno F, Hamilton H, Brownell RL, Cao Y, Ding W, Zuoyan Z, Shedlock AM, Fordyce RE, Hasegawa M, Okada N: Retroposon analysis of major cetacean lineages: the monophyly of toothed whales and the paraphyly of river dolphins. Proc Natl Acad Sci USA 2001, 98:7384-7389.

40. Kawai K, Nikaido M, Harada M, Matsumura S, Lin LK, Wu Y, Hasegawa M, Okada N: Intra- and interfamily relationships of Vespertilionidae inferred by various molecular markers including SINE insertion data. J Mol Evol 2002, 55:284-301.

41. Sasaki T, Takahashi K, Nikaido M, Miura S, Yasukawa Y, Okada N: First application of the SINE (short interspersed repetitive element) method to infer phylogenetic relationships in reptiles: an example from the turtle superfamily Testudinoidea. Mol Biol Evol 2004, 21:705-715.

42. Nikaido M, Hamilton H, Makino H, Sasaki T, Takahashi K, Goto M, Kanda N, Pastene LA, Okada N: Baleen whale phylogeny and a past extensive radiation event revealed by SINE insertion analysis. Mol Biol Evol 2006, 23:866-873.

43. Nishihara $\mathrm{H}$, Smit AF, Okada N: Functional noncoding sequences derived from SINEs in the mammalian genome. Genome Res 2006, 16:864-874.

44. Joly S, Bruneau A: Incorporating allelic variation for reconstructing the evolutionary history of organisms from multiple genes: An example from Rosa in North America. Syst Biol 2006, 55:623-636.

45. Ané C, Larget B, Baum DA, Smith SD, Rokas A: Bayesian estimation of concordance among gene trees. Mol Phylogenet Evol 2007, 24:412-426.

46. Rokas A, Carroll S: Bushes in the tree of life. Plos Biol 2006, 4:1899-1904.

47. Baryshnikov GF, Abramov AV: Structure of baculum (os penis) in Mustelidae (Mammalia, Carnivora). Communication 1. Zoo Zh 1997, 76:1399-1410, in Russian.

48. Baryshnikov GF, Abramov AV: Structure of baculum (os penis) in Mustelidae (Mammalia, Carnivora). Communication 2. Zoo Zh 1998, 77:231-236, in Russian.

49. Nie W, Wang J, O'Brien PCM, Fu B, Ying T, Ferguson-Smith MA, Yang F: The genome phylogeny of domestic cat, red panda and five mustelid species revealed by comparative chromosome painting and G-banding. Chromosome Res 2002, 10:209-222.

50. Durando CM, Baker RH, Etges WJ, Heed EW, Wasserman M, Desalle R: Phylogenetic analysis of the repleta species group of the Genus Drosophila using multiple sources of characters. Mol Phylogenet Evol 2000, 16:296-307.

51. Goto SG, Kimura MT: Phylogenetic utility of mitochondrial $\mathrm{CO}$ and nuclear Gpdh genes in Drosophila. Mol Phylogenet Evol 2001, 18:404-422.

52. Giannasi N, Malhotra A, Thorpe RS: Nuclear and mtDNA phylogenies of the Trimeresurus complex: implications for the gene versus species tree debate. Mol Phylogenet Evol 2001, 19:57-66.

53. Yu L, Li QW, Ryder OA, Zhang YP: Phylogeny of the bears (Ursidae) based on nuclear and mitochondrial genes. Mol Phylogenet Evol 2004, 32:480-494.

54. Nei M: Molecular evolutionary genetics. New York: Columbia University Press; 1987.

55. Pamilo P, Nei M: Relationships between gene trees and species trees. Mol Biol Evol 1988, 4:526-543.

56. Wu C-1: Inferences of species phylogeny in relation to segregation of ancient polymorphisms. Genetics 1991, 127:429-435.

57. Moore WS: Inferring phylogenies from mtDNA variation: Mitochondrial gene trees versus nuclear gene trees. Evolution 1995, 49:718-726. 
58. Bininda-Emonds ORP, Gittleman $J$, Purvis A: Building large trees by combining phylogenetic information: A complete phylogeny of the extant Carnivora (Mammalia). Biol Rev 1999, 74:143-175.

59. Eizirik E, Murphy WJ, Koepfli KP, Johnson WE, Dragoo JW, Wayne RK, O'Brien SJ: Pattern and timing of diversification of the mammalian order Carnivora inferred from multiple nuclear gene sequences. Mol Phylogenet Evol 2010, 56:49-63.

60. Steininger FF: Chronostratigraphy, geochronology and biochronology of the Miocene "European Land Mammal Mega-Zones" (ELMMZ) and the Miocene "Mammal-Zones (MN-Zones)". In The Miocene Land Mammals of Europe. Edited by: Rossner GE, Heissig K. Munich: Verlag Dr. Friedrich Pfeil; 1999:9-24

61. Schlunegger F, Burbank DW, Matter A, Engesser B, Modden C: Magnetostraitigraphic calibration of the Oligocene to Middle Miocene (30-15 Ma) mammal biozones and depositional sequences of the Swiss Molasse Basin. Eclogae Geol Helv 1996, 89:753-788.

62. Morlo M, Kundrát M: The first carnivoran fauna from the Ruscinium (Early Pliocene, MN 15) of Germany. Paléontol Z 2001, 75:163-187.

63. Hosoda T, Suzuki H, Harada M, Tsuchiya K, Han S-H, Zhang Y-P, Kryukov AP, Lin L-K: Evolutionary trends of the mitochondrial lineage differentiation in species of genera Martes and Mustela. Genes Genet Syst 2000, 75:259-267.

64. Wayne RK, Benveniste RE, Janczewski DN, O'Brien SJ: Molecular and biochemical evolution of the Carnivora. In Carnivore Behavior, Ecology, and Evolution. Edited by: Gittleman JL. New York: Cornell University Press; 1989:

65. Glazek J, Sulimski A, Wysoczanski-Minkowicz T: On the stratigraphic position of Weze 1 locality (Middle Poland). In Proceedings of the 6th International Congress of Speleology, Olomouc-CSSR. Volume 1. Prague; 1976:435-442.

66. Glazek J, Szynkiewicz A: Kras. In Przewodnik LIl Zjazdu Polskiego Towarzystwa Geologicznego, Belchatow, 11-14 wrzesnia 1980. Edited by: Barczyk WW Wydawnictwa Geologiczne; 1980:242-267.

67. Möller-Krull M, Delsuc F, Churakov G, Marker C, Superina M, Brosius J, Douzery EJ, Schmitz J: Retroposed elements and their flanking region resolve the evolutionary history of xenarthran mammals (armadillos, anteaters, and sloths). Mol Biol Evol 2007, 24:2573-2582.

68. Benavides E, Baum R, McClellan D, Sites JW: Molecular phylogenetics of the lizard genus Microlophus (squamata:tropiduridae): aligning and retrieving indel signal from nuclear introns. Syst Biol 2007, 56:776-797.

69. Zardoya R, Meyer A: Phylogenetic performance of mitochondrial proteincoding genes in resolving relationships among vertebrates. Mol Biol Evol 1996, 13:933-942.

70. Russo CAM, Takezaki N, Masatoshi N: Efficiencies of different genes and different tree-building methods in recovering a known vertebrate phylogeny. Mol Biol Evol 1996, 13:525-536.

71. Miya M, Nishida M: Use of mitogenomic information in teleostean molecular phylogenetics: a tree-based exploration under the maximum parsimony optimality criterion. Mol Phylogenet Evol 2000, 17:437-455.

72. Mueller RL: Evolutionary rates, divergence dates, and the performance of mitochondrial genes in Bayesian phylogenetic analysis. Syst Biol 2006, 55:289-300.

73. Yu L, Li YW, Ryder OA, Zhang YP: Analysis of complete mitochondrial genome sequences increases phylogenetic resolution of bears (Ursidae), a mammalian family that experienced rapid speciation. BMC Evol Biol 2007, 7:198.

74. Cunha RL, Grande C, Zardoya R: Neogastropod phylogenetic relationships based on entire mitochondrial genomes. BMC Evol Biol 2009, 9:210.

75. Creer S: Choosing and using introns in molecular phylogenetics. Evol Bioinform 2007, 3:99-108.

76. Yu L, Li QW, Ryder OA, Zhang YP: Phylogenetic relationships within mammalian order Carnivora indicated by sequences of two nuclear DNA genes. Mol Phylogenet Evol 2004, 33:694-705.

77. Yu L, Zhang YP: Phylogeny of the caniform carnivora: evidence from multiple genes. Genetica 2006, 127:65-79.

78. Thompson JD, Gibson TJ, Plewniak F, Jeanmougin F, Higgins DG: The clustalx windows interface: flexible strategies for multiple sequence alignment aided by quality analysis tools. Nucleic Acids Res 1997, 24:4876-4882

79. Castresana J: Selection of conserved blocks from multiple alignments for their use in phylogenetic analysis. Mol Biol Evol 2000, 17:540-552.
80. Tamura K, Dudley J, Nei M, Kumar S: MEGA4: Molecular Evolutionary Genetics Analysis (MEGA) software version 4.0. Mol Biol Evol 2007, 24:1596-1599.

81. Yu L, Zhang YP: Evolutionary implications of multiple SINE insertions in an intronic region from diverse mammals. Mamm Genome 2005, 16:651-660.

82. Swofford DL: PAUP*: Phylogenetic analysis using parsimony (* and Other Methods), Version 4. Sinauer Associates, Sunderland, Massachusetts; 2002.

83. Ronquist F, Huelsenbeck JP: MrBayes 3: Bayesian phylogenetic inference under mixed models. Bioinformatics 2003, 19:1572-1574.

84. Akaike $\mathrm{H}$ : A new look at the statistical model identification. IEEE Trans Autom contr 1974, 19:716-723.

85. Posada D, Buckley TR: Model selection and model averaging in phylogenetics: advantages of akaike information criterion and bayesian approaches over likelihood ratio tests. Syst Biol 2004, 53:793-808.

86. Posada D, Crandall KA: MODELTEST: testing the model of DNA substitution. Bioinformatics 1998, 14:817-818.

87. Felsenstein J: Confidence limits on phylogenies: An approach using the bootstrap. Evolution 1985, 39:783-791.

88. Stamatakis A, Hoover $\mathrm{P}$, Rougemont J: A rapid bootstrap algorithm for the RAxML Web servers. Syst Biol 2008, 57:758-771.

89. Larget B, Simon DL: Markov chain Monte Carlo algorithms for the Bayesian analysis of phylogenetic trees. Mol Biol Evol 1999, 16:750-759.

90. Joly S, Bruneau A: Incorporating allelic variation for reconstructing the evolutionary history of organisms from multiple genes: An example from Rosa in North America. Syst Biol 2006, 55:623-636.

91. Drummond AJ, Rambaut A: BEAST: Bayesian evolutionary analysis by sampling trees. BMC Evol Biol 2007, 7:214.

92. Luterbacher HP, Ali JR, Brinkhuis H, Gradstein FM, Hooker JJ, Monechi S, Ogg JG, Powell J, Röhl U, Sanfilippo A, Schmitz B: The Paleogene Period. In A Geologic Time Scale. Edited by: Gradstein FM, Ogg JG, Smith AG. Cambridge: Cambridge University Press; 2004:384-408.

93. Fortelius M, Werdelin L, Andrews P, Bernor RL, Gentry A, Humphrey L, Mittmann H-W, Viratana S: Provinciality, diversity, turnover, and paleoecology on land mammal faunas of the later Miocene of Western Eurasia. In The evolution of western Eurasian Neogene mammal faunas. Edited by: Bernor RL, Fahlbusch V, Mittmann H-V. New York: Columbia University Press; 1996:

94. Rambaut A, Drummond AJ: Tracer v1.5. 2007 [http://beast.bio.ed.ac.uk/ Tracer].

95. Shimodaira H, Hasegawa M: Multiple Comparisons of Log-Likelihoods with Applications to Phylogenetic Inference. Mol Biol Evol 1999, 6:1114-1116.

96. Shimodaira $\mathrm{H}$ : An approximately unbiased test of phylogenetic tree selection. Syst Biol 2002, 51:492-508.

97. Shimodaira $H$, Hasegawa M: CONSEL: for assessing the confidence of phylogenetic tree selection. Bioinformatics 2001, 17:1246-1247.

doi:10.1186/1471-2148-11-92

Cite this article as: Yu et al:: On the phylogeny of Mustelidae subfamilies: analysis of seventeen nuclear non-coding loci and mitochondrial complete genomes. BMC Evolutionary Biology 2011 11:92.

\section{Submit your next manuscript to BioMed Central and take full advantage of:}

- Convenient online submission

- Thorough peer review

- No space constraints or color figure charges

- Immediate publication on acceptance

- Inclusion in PubMed, CAS, Scopus and Google Scholar

- Research which is freely available for redistribution

Submit your manuscript at www.biomedcentral com/submit
C Biomed Central 